\title{
Interpolation on algebraic groups
}

\author{
S. Fischler
}

\begin{abstract}
We prove an interpolation lemma with multiplicities on a commutative algebraic group. This statement is 'dual' to zero estimates used in transcendental number theory. The special case where no multiplicities are involved has been proved by Masser.
\end{abstract}

\section{Introduction}

The usual sketch of a proof of transcendance or algebraic independence is to construct an 'auxiliary function' which takes small algebraic values at many given points; these values are then proved to be zero. The final step is to prove that such a function, vanishing at these points, has to be zero: such a statement is called a zero estimate.

Another 'dual' approach is to construct an 'auxiliary functional', i.e. a linear combination of evaluations of derivations, which takes small algebraic values (and therefore vanishes) on many functions (see [Wal91] or [Wal00]). In this case, the final step (i.e., proving the auxiliary functional is zero) is achieved thanks to an interpolation lemma.

In the context of commutative algebraic groups (e.g. $\mathbb{G}_{a}, \mathbb{G}_{m}$, elliptic curves, abelian varieties, ...), such an interpolation lemma has been proved by Masser [Mas82] when no multiplicities are involved. The present paper provides a proof in the general case.

This interpolation lemma can be used to provide new proofs of many known results in transcendental number theory, for instance [Wal89] Schneider's theorem on transcendance of elliptic integrals of the first kind. This may allow one to sharpen some quantitative statements. Other applications of this interpolation lemma to transcendental number theory may appear (see [Wal82]).

The proof given below follows the same lines as Masser's. First, the statement is translated in terms of functionals ( $\S 1.5$ ). Then a special 'non-degenerate' case is studied ( $\S 4.1$ ), using a zero estimate. Finally, the general case is deduced ( $\S 4.2)$ by induction upon the dimension of the algebraic group $G$.

The two main tools used in the proof are dealt with in $\S \S 2$ and 3 . The first one is how to handle operations on functionals (projection on a quotient, translation, derivation); the Baker-CoatesAnderson trick is used in a crucial way. The second tool is a general study of obstructing subgroups to interpolation, and to zero estimates, which replaces the use of distribution exponents [Wal79] which suffices in Masser's setting.

\subsection{Statement of the theorem}

In this text, we let $\mathbb{N}=\{0,1,2, \ldots\}$ denote the set of all non-negative integers.

Let $G$ be a connected commutative algebraic group, equipped with a locally closed immersion into $\mathbb{P}^{N}$ (corresponding to the choice of a very ample divisor on a compactification of $G$ ).

Received 16 September 2003, accepted in final form 7 August 2004, published online 21 June 2005. 2000 Mathematics Subject Classification 14L10 (primary), 11J95, 14L40, 14K25 (secondary).

Keywords: algebraic group, interpolation, zero estimate.

This journal is (c) Foundation Compositio Mathematica 2005. 


\section{S. FISCHLER}

The base field, in the whole text, is $\mathbb{C}$ (though any algebraically closed field of characteristic zero could be considered, for instance its $p$-adic analog $\mathbb{C}_{p}$; see also [Mas82, $\left.\S 1\right]$ ).

Let us denote by $n$ the dimension of $G$, and by $T G$ the tangent space to $G$ at 1 . We identify $T G$ with the space of translation-invariant vector fields on $G$.

For $D \geqslant 0$, we let $\mathcal{R}(G)_{D}=H^{0}(\bar{G}, \mathcal{O}(D))$ be the vector space of global sections of the line bundle $\mathcal{O}(D)$ on the Zariski closure $\bar{G}$ of $G$ in $\mathbb{P}^{N}$. By abuse of language, we shall call such a global section homogeneous polynomial of degree $D$. We let also $\mathcal{R}(G)=\bigoplus_{D \geqslant 0} \mathcal{R}(G)_{D}$; this is a graded algebra, the elements of which are called polynomials with the same abuse.

Let $\Gamma$ be a finitely generated subgroup of $G(\mathbb{C})$, spanned by $\left(\gamma_{1}, \ldots, \gamma_{l}\right)$ with $l \geqslant 0$. For $S_{1}, \ldots, S_{l} \in \mathbb{R}_{>0}$ we let $\Gamma\left(S_{1}, \ldots, S_{l}\right)$ denote the set of all linear combinations $n_{1} \gamma_{1}+\cdots+n_{l} \gamma_{l}$, with integers $n_{j}$ such that $\left|n_{j}\right|<S_{j}$ for all $j \in\{1, \ldots, l\}$. We assume (without loss of generality: see [MW81, p. 492])

$$
\Gamma \subset\left\{X_{0} \neq 0\right\} \subset \mathbb{P}^{N} .
$$

Let $W$ be a subspace of $T G$, of dimension $d \geqslant 0$, and let $\left(\partial_{1}, \ldots, \partial_{d}\right)$ be a basis of $W$. For a family $\underline{T}=\left(T_{1}, \ldots, T_{d}\right)$ of $d$ positive real numbers, we let $\mathbb{N}_{\underline{T}}^{d}$ be the set of all $\sigma=\left(\sigma_{1}, \ldots, \sigma_{d}\right) \in \mathbb{N}^{d}$ such that $\sigma_{j}<T_{j}$ for all $j \in\{1, \ldots, d\}$.

We denote by $\mathrm{Op}_{W}$ the set of all polynomials in $\partial_{1}, \ldots, \partial_{d}$, i.e. the space of differential operators along $W$. We denote by $\mathrm{Op}_{\underline{\partial}, \underline{T}}$ the subspace of $\mathrm{Op}_{W}$ spanned by the monomials $\partial^{\sigma}=\partial_{1}^{\sigma_{1}} \ldots \partial_{d}^{\sigma_{d}}$ for $\sigma \in \mathbb{N}_{T}^{d}$. We say that a polynomial $P \in \mathcal{R}(G)_{D}$ vanishes up to order $\underline{T}$ along $W$ at a point $\gamma \in \Gamma$ if $\partial^{\sigma}\left(P / X_{0}^{D}\right)(\gamma)=0$ for any $\sigma \in \mathbb{N}_{\underline{T}}^{d}$. Of course, this depends on the basis $\left(\partial_{1}, \ldots, \partial_{d}\right)$, and not only on $W$.

The main result of this paper is the following theorem, which is best possible up to the value of $c_{1}$ (see the end of $\S 1.5$ ).

Theorem 1.1. There is a positive constant $c_{1}$, depending on all previous data, with the following property. Let $D, S_{1}, \ldots, S_{l}, T_{1}, \ldots, T_{d}$ be positive integers such that, for any non-zero connected algebraic subgroup $H$ of $G$, we have

$$
\#(H \cap \Gamma(\underline{S})) \operatorname{dim}\left(\mathrm{Op}_{W \cap T H} \cap \mathrm{Op}_{\underline{\partial}, \underline{T}}\right)<c_{1} D^{\operatorname{dim}(H)} .
$$

For all $\gamma \in \Gamma(\underline{S})$ and $\sigma \in \mathbb{N}_{T}^{d}$, let $a_{\gamma, \sigma}$ be a complex number. Then there exists $P \in \mathcal{R}(G)$, homogeneous of degree $D$, such that $\partial^{\sigma}\left(P / X_{0}^{D}\right)(\gamma)=a_{\gamma, \sigma}$ for all $\gamma \in \Gamma(\underline{S})$ and $\sigma \in \mathbb{N}_{\underline{T}}^{d}$.

Remarks. If we consider, instead of $\Gamma(\underline{S})$, the set of points $n_{1} \gamma_{1}+\cdots+n_{l} \gamma_{l}$ with $0 \leqslant n_{j}<S_{j}$, then the assumption in Theorem 1.1 has to be made with every translate of any algebraic subgroup $H$ of $G$.

If the group $G$ is linear (i.e., isomorphic to $\mathbb{G}_{a}^{\ell_{0}} \times \mathbb{G}_{m}^{\ell_{1}}$ for some $\ell_{0}, \ell_{1} \geqslant 0$ ), Theorem 1.1 can be deduced from a zero estimate using Fourier-Borel transform (see [Wal91] or [Wal00]). This is written, using the language of Hopf algebras, in [Fis03, Chapter 8]. The interpolation lemma proved in this way is even more precise: bi-degrees $\left(D_{0}, D_{1}\right)$ can be considered (with respect to the additive and multiplicative parts of the group), instead of just $D$.

Masser's interpolation lemma is the special case of Theorem 1.1 where no multiplicities are involved (i.e. $T_{1}=\cdots=T_{d}=1$ or $\operatorname{dim}(W)=0$ ), and equality $S_{1}=\cdots=S_{l}$ is assumed. Therefore, even without multiplicities, Theorem 1.1 is more precise ${ }^{1}$ than Masser's interpolation lemma. Theorem 1.1 should be enough for applications to transcendental number theory, but it would be interesting to refine it anyway and obtain interpolation lemmas in the same setting as zero estimates (see [Phi86]).

${ }^{1}$ An intermediate statement, namely Theorem 1.1 under the assumptions $T_{1}=\cdots=T_{d}$ and $S_{1}=\cdots=S_{l}$, is proved in [Fis03, Chapter 7]. 


\section{INTERPOLATION ON ALGEBRAIC GROUPS}

\subsection{Cohomological interpretation}

In this subsection, we state (following a suggestion of Pascal Autissier) Theorem 1.1 in terms of vanishing of a cohomology module $H^{1}$. This is the reason why Bertrand [Ber04] uses the terminology 'ampleness lemma' instead of 'interpolation lemma'.

For positive integers $\underline{S}=\left(S_{1}, \ldots, S_{l}\right)$ and $\underline{T}=\left(T_{1}, \ldots, T_{d}\right)$, let $\mathcal{J}_{\underline{S}, \underline{T}}$ be the ideal sheaf whose sections, on any open subset $U$ of $\bar{G}$, are given by

$$
\Gamma\left(U, \mathcal{J}_{\underline{S}}, \underline{T}\right)=\left\{P \in \mathcal{O}_{U}, P \text { vanishes up to order } \underline{T} \text { along } W \text { at each point of } \Gamma(\underline{S}) \cap U\right\} .
$$

Let $Y_{\underline{S}, \underline{T}}$ be the corresponding closed subscheme of $\bar{G}$; it consists of all points in $\Gamma(\underline{S})$, thickened up to order $\underline{T}$ along $W$. For any non-negative integer $D$, let $\mathcal{I}_{\underline{S}, \underline{T}, D}=\mathcal{J}_{\underline{S}, \underline{T}} \otimes \mathcal{O}(D)$.

We have a short exact sequence of $\mathcal{O}_{\bar{G}}$-modules

$$
0 \rightarrow \mathcal{J}_{\underline{S}, \underline{T}} \rightarrow \mathcal{O}_{\bar{G}} \rightarrow i_{*} \mathcal{O}_{Y_{\underline{S}, \underline{T}}} \rightarrow 0
$$

where $i$ is the inclusion $Y_{\underline{S}, \underline{T}} \hookrightarrow \bar{G}$. Tensoring by $\mathcal{O}(D)$ yields an exact sequence

$$
0 \rightarrow \mathcal{I}_{\underline{S}, \underline{T}, D} \rightarrow \mathcal{O}_{\bar{G}}(D) \rightarrow k_{\underline{S}, \underline{T}, D} \rightarrow 0,
$$

and hence a long exact sequence of cohomology

$$
0 \rightarrow H^{0}\left(\bar{G}, \mathcal{I}_{\underline{S}, \underline{T}, D}\right) \rightarrow H^{0}\left(\bar{G}, \mathcal{O}_{\bar{G}}(D)\right) \stackrel{\alpha}{\rightarrow} H^{0}\left(\bar{G}, k_{\underline{S}, \underline{T}, D}\right) \rightarrow H^{1}\left(\bar{G}, \mathcal{I}_{\underline{S}, \underline{T}, D}\right) \rightarrow 0
$$

when $D$ is large enough in terms of $G$, because in this case $H^{1}\left(\bar{G}, \mathcal{O}_{\bar{G}}(D)\right)=0$ ([Har77, p. 228]). Therefore $\alpha$ is surjective if and only if $H^{1}\left(\bar{G}, \mathcal{I}_{\underline{S}, \underline{T}, D}\right)=0$ (when $D$ is large enough). This allows us to translate Theorem 1.1 in the following way

Theorem 1.2. There is a positive constant $c_{2}$ with the following property. Let $D, S_{1}, \ldots, S_{l}$, $T_{1}, \ldots, T_{d}$ be positive integers such that, for any non-zero connected algebraic subgroup $H$ of $G$, we have

$$
\#(H \cap \Gamma(\underline{S})) \operatorname{dim}\left(\mathrm{Op}_{W \cap T H} \cap \mathrm{Op}_{\underline{\partial}, \underline{T}}\right)<c_{2} D^{\operatorname{dim}(H)} .
$$

Then $H^{1}\left(\bar{G}, \mathcal{I}_{\underline{S}, \underline{T}, D}\right)=0$.

Remark. If $G$ is an abelian variety then $H^{1}\left(\bar{G}, \mathcal{O}_{\bar{G}}(D)\right)=0$ for any $D \geqslant 1$, and one can take $c_{2}=c_{1}$.

\subsection{Translation in terms of functionals}

Let $\mathbb{C} \Gamma$ denote the group algebra of $\Gamma$, consisting of formal finite linear combinations (over $\mathbb{C}$ ) of elements of $\Gamma$. Let $\operatorname{Sym}(W)=\bigoplus_{k \geqslant 0} \operatorname{Sym}^{k} W$ denote the symmetric algebra of $W$. We let

$$
\mathcal{F}=\mathbb{C} \Gamma \otimes \operatorname{Sym}(W)
$$

and call functional any element of $\mathcal{F}$ that is any finite linear combination of elements of the shape $\gamma \otimes\left(\partial^{(1)} \ldots \ldots \partial^{(k)}\right)$ with $\gamma \in \Gamma$ and $\partial^{(1)}, \ldots, \partial^{(k)} \in W$. In case any confusion may arise, we will write $\mathcal{F}^{\Gamma, W}$ instead of $\mathcal{F}$. In the whole text, $\mathcal{F}$ is considered as a vector space, and never as an algebra.

A functional $\eta \in \mathcal{F}$ can be evaluated on a polynomial $P \in \mathcal{R}(G)_{D}$ : by linearity, it suffices to define this evaluation when $\eta=\gamma \otimes\left(\partial^{(1)} \ldots \partial^{(k)}\right)$, and in this case we let

$$
\eta(P)=\partial^{(1)} \cdots \partial^{(k)}\left(\frac{P(\underline{X})}{X_{0}^{D}}\right)(\gamma)
$$

This makes sense thanks to (1).

Fix a basis $\left(\partial_{1}, \ldots, \partial_{d}\right)$ of $W$. Then a basis of $\mathcal{F}=\mathcal{F}^{\Gamma, W}$ is given by the functionals ev $\gamma, \sigma=\gamma \otimes \partial^{\sigma}$ for $\gamma \in \Gamma$ and $\sigma \in \mathbb{N}^{d}$, where $\partial^{\sigma}=\partial_{1}^{\sigma_{1}} \cdots \partial_{d}^{\sigma_{d}}$. Accordingly, $\mathcal{F}$ is isomorphic to the $\mathbb{C}$-algebra of the semi-group $\Gamma \times \mathbb{N}^{d}$. Moreover, the evaluation of $\eta=\sum_{\gamma, \sigma} \lambda_{\gamma, \sigma} \operatorname{ev}_{\gamma, \sigma} \in \mathcal{F}$ on $P \in \mathcal{R}(G)_{D}$ is 


\section{S. FischleR}

given by

$$
\eta(P)=\sum_{\gamma, \sigma} \lambda_{\gamma, \sigma} \partial^{\sigma}\left(\frac{P}{X_{0}^{D}}\right)(\gamma) .
$$

We define a filtration on $\mathcal{F}$ by letting, for non-negative integers $S_{1}, \ldots, S_{l}$ and $T_{1}, \ldots, T_{d}$

$$
\mathcal{F}_{\underline{S}, \underline{T}}=\operatorname{Span}\left\{\operatorname{ev}_{\gamma, \sigma} ; \gamma \in \Gamma(\underline{S}), \sigma \in \mathbb{N}_{\underline{T}}^{d}\right\} .
$$

The way $\mathcal{F}$ is defined, and acts on $\mathcal{R}(G)$, depends neither on the choice of a basis of $W$, nor on the choice of a generating set for $\Gamma$. However, $\mathcal{F}_{\underline{S}, \underline{T}}$ does depend on the choice of $\gamma_{1}, \ldots, \gamma_{l}$ and $\partial_{1}, \ldots, \partial_{d}$. For any finite subset $\Sigma$ of $\mathbb{P}^{N}(\mathbb{C})$, contained in the open set $\left\{X_{0} \neq 0\right\}$, we let $\mathcal{F}^{\Sigma, W}=\left(\operatorname{Span}_{\mathbb{C}} \Sigma\right) \otimes \operatorname{Sym}(W)$, where the linear combinations of elements of $\Sigma$ are understood formally. The space $\mathcal{F}^{\Sigma, W}$ is filtered by the subspaces $\mathcal{F}_{\underline{T}}^{\Sigma, W}$, defined for non-negative integers $\underline{T}$ by

$$
\mathcal{F}_{\underline{T}}^{\Sigma, W}=\left(\operatorname{Span}_{\mathbb{C}} \Sigma\right) \otimes\left(\operatorname{Span}\left\{\partial^{\sigma}, \sigma \in \mathbb{N}_{\underline{T}}^{d}\right\}\right) .
$$

In what follows, $D, S_{1}, \ldots, S_{l}$ and $T_{1}, \ldots, T_{d}$ are real numbers (not always integers) with $D \geqslant 0$ and $\underline{S}, \underline{T} \geqslant 1$. We let $\mathcal{R}(G)_{D}$ be the space of homogeneous polynomials on $G$, of degree $[D]$ (the integer part of $D$ ), and $\mathcal{F}_{\underline{S}, \underline{T}}=\mathcal{F}_{\left\lceil S_{1}\right\rceil, \ldots,\left\lceil S_{l}\right\rceil,\left\lceil T_{1}\right\rceil, \ldots,\left\lceil T_{d}\right\rceil}$, in such a way that (3) is satisfied even if the components of $\underline{S}$ and $\underline{T}$ are not integers (here $\lceil x\rceil$ is the least integer greater than or equal to $x)$.

The crucial role played by functionals in this setting is explained by the following lemma, which is used to translate Theorem 1.1 into Theorem 1.7 stated below.

Lemma 1.3. Let $D \geqslant 0$ and $S_{1}, \ldots, S_{l}, T_{1}, \ldots, T_{d} \geqslant 1$ be real numbers. The following are equivalent:

(i) for any complex numbers $a_{\gamma, \sigma}$, indexed by $\gamma \in \Gamma(\underline{S})$ and $\sigma \in \mathbb{N}_{\underline{T}}^{d}$, there is a polynomial $P \in \mathcal{R}(G)_{D}$ such that $\partial^{\sigma}\left(P / X_{0}^{D}\right)(\gamma)=a_{\gamma, \sigma}$ for all these $\gamma, \sigma$;

(ii) there is no non-zero functional $\eta \in \mathcal{F}_{\underline{S}, \underline{T}}$ such that $\eta(P)=0$ for all $P \in \mathcal{R}(G)_{D}$.

Proof. Consider the linear map from $\mathcal{R}(G)_{D}$ to $\mathbb{C}^{\Gamma(\underline{S}) \times \mathbb{N}_{T}^{d}}$ which associates to each polynomial $P$ the family of values $\partial^{\sigma}\left(P / X_{0}^{D}\right)(\gamma)$. The first statement means the map is surjective; the second one means the image of this map is contained in no hyperplane.

Remark. For $P \in \mathcal{R}(G)_{D}$ and $k \in \mathbb{N}$, we have $\eta(P)=\eta\left(X_{0}^{k} P\right)$ for all $\eta \in \mathcal{F}$. Accordingly, if $\eta \in \mathcal{F}_{\underline{S}, \underline{T}}$ vanishes identically on $\mathcal{R}(G)_{D}$, then for all $\underline{S}^{\prime} \geqslant \underline{S}, \underline{T}^{\prime} \geqslant \underline{T}$ and $D^{\prime} \leqslant D$ we have $\eta \in \mathcal{F}_{\underline{S}^{\prime}, \underline{T}^{\prime}}$ and $\eta$ vanishes identically on $\mathcal{R}(G)_{D^{\prime}}$.

\subsection{Some counting lemmas}

In what follows, when a group $\Gamma$ is equipped with a system $\left(\gamma_{1}, \ldots, \gamma_{l}\right)$ of generators, we denote by $\Gamma_{j}$ the subgroup of $\Gamma$ generated by $\gamma_{1}, \ldots, \gamma_{j}$, for $j \in\{0, \ldots, l\}$ (hence $\Gamma_{0}=\{0\}$ ). The following lemma will be useful (see [Mas82, Lemma 3]).

Lemma 1.4. Let $\Gamma$ and $\Gamma^{\prime}$ be subgroups of $G(\mathbb{C})$, equipped with generators $\left(\gamma_{1}, \ldots, \gamma_{l}\right)$ and $\left(\gamma_{1}^{\prime}, \ldots, \gamma_{l^{\prime}}^{\prime}\right)$. Assume there are $0 \leqslant k_{1} \leqslant \cdots \leqslant k_{l} \leqslant l^{\prime}$ such that $\Gamma_{j} \subset \Gamma_{k_{j}}^{\prime}$ for all $j \in\{1, \ldots, l\}$. Then there is a constant $c_{3}$, depending only on $\gamma_{1}, \ldots, \gamma_{l}$ and on $\gamma_{1}^{\prime}, \ldots, \gamma_{l^{\prime}}^{\prime}$, such that for any $S_{1} \geqslant \cdots \geqslant S_{l} \geqslant 1$ we have

$$
\Gamma\left(S_{1}, \ldots, S_{l}\right) \subset \Gamma^{\prime}\left(c_{3} S_{1}^{\prime}, \ldots, c_{3} S_{l^{\prime}}^{\prime}\right),
$$

where $S_{k}^{\prime}=S_{j}$ for $k_{j-1}<k \leqslant k_{j}$ and $1 \leqslant j \leqslant l+1$, with $k_{0}=0, k_{l+1}=l^{\prime}$ and $S_{l+1}=1$.

Proof. There are integers $c_{k, j}$, for $j \in\{1, \ldots, l\}$ and $k \leqslant k_{j}$, such that $\gamma_{j}=\sum_{k=1}^{k_{j}} c_{k, j} \gamma_{k}^{\prime}$. Let $M$ denote the maximum of all absolute values $\left|c_{k, j}\right|$. Let $\gamma=\sum_{j=1}^{l} s_{j} \gamma_{j}$ belong to $\Gamma(\underline{S})$, 


\section{INTERPOLATION ON ALGEBRAIC GROUPS}

with $\left|s_{j}\right|<S_{j}$. Then $\gamma=\sum_{k=1}^{k_{l}} s_{k}^{\prime} \gamma_{k}^{\prime}$ with $s_{k}^{\prime}=\sum_{j} s_{j} c_{k, j}$ (the sum is over all $j$ such that $k_{j} \geqslant k$ ). Hence $\left|s_{k}^{\prime}\right| \leqslant M l S_{k}^{\prime}$, thereby proving the lemma.

Lemma 1.5. Let $\Gamma$ be a subgroup of $G(\mathbb{C})$, equipped with a family $\left(\gamma_{1}, \ldots, \gamma_{l}\right)$ of generators. Let $H$ be an algebraic subgroup of $G$. Then there are two positive constants $c_{4}$ and $c_{5}$, depending on $\gamma_{1}, \ldots, \gamma_{l}$ and on $H$, such that for all real numbers $S_{1} \geqslant \cdots \geqslant S_{l} \geqslant 1$ we have

$$
c_{4} \prod_{j=1}^{l} S_{j}^{\operatorname{rk}\left(\frac{\Gamma_{j} \cap H}{\Gamma_{j-1} \cap H}\right)}<\#(H \cap \Gamma(\underline{S}))<c_{5} \prod_{j=1}^{l} S_{j}^{\operatorname{rk}\left(\frac{\Gamma_{j} \cap H}{\Gamma_{j-1} \cap H}\right)},
$$

where $\Gamma_{j}$ is the subgroup of $\Gamma$ generated by $\gamma_{1}, \ldots, \gamma_{j}$.

Proof. Let $1 \leqslant j_{1}<\cdots<j_{r} \leqslant l$ be the indices $j$ such that $\operatorname{rk}\left(\left(\Gamma_{j} \cap H\right) /\left(\Gamma_{j-1} \cap H\right)\right)=1$. Consider the subgroups $\Gamma_{j_{1}} \cap H \subset \cdots \subset \Gamma_{j_{r}} \cap H$. As $\operatorname{rk}\left(\Gamma_{j_{t}} \cap H\right)=t$ for any $t \in\{1, \ldots, r\}$, it is possible to find $\alpha_{1}, \ldots, \alpha_{r} \in \Gamma$ such that the subgroup generated by $\alpha_{1}, \ldots, \alpha_{t}$ is contained in $\Gamma_{j_{t}} \cap H$, and of finite index, for any $t \in\{1, \ldots, r\}$. Lemma 1.4 gives a constant $c_{3}$, independent of $\underline{S}$, such that $\Gamma(\underline{S}) \cap H$ contains $A\left(S_{j_{1}} / c_{3}, \ldots, S_{j_{r}} / c_{3}\right)$, where $A$ is the subgroup of $\Gamma$ generated by $\alpha_{1}, \ldots, \alpha_{r}$. This concludes the proof of the lower bound, since $\alpha_{1}, \ldots, \alpha_{r}$ are linearly independent over $\mathbb{Z}$.

To prove the upper bound, we use induction on $l$; for $l=0$ the statement is obvious. Assume it is true for $\Gamma_{l-1}$, which is generated by $\gamma_{1}, \ldots, \gamma_{l-1}$. Notice that $\Gamma(\underline{S})$ is the union, for $|n|<S_{l}$, of the set $n \gamma_{l}+\Gamma_{l-1}\left(S_{1}, \ldots, S_{l-1}\right)$. This proves the result if $\operatorname{rk}\left(\left(\Gamma_{l} \cap H\right) /\left(\Gamma_{l-1} \cap H\right)\right)=1$. Let us assume now that $\Gamma_{l-1} \cap H$ has finite index, say $N$, in $\Gamma \cap H$. Let $\beta_{1}, \ldots, \beta_{N} \in \Gamma \cap H$ be representatives of the cosets; write $\beta_{i}=\sum_{j=1}^{l} \lambda_{i, j} \gamma_{j}$ for any $i \in\{1, \ldots, N\}$. Let $K$ be the smallest integer $k \geqslant 1$ such that $k \gamma_{l} \in \Gamma_{l-1}$, with $K=\infty$ if there is no such $k$. If $K$ is finite, let $a_{1}, \ldots, a_{l-1}$ be such that $K \gamma_{l}=\sum_{j=1}^{l-1} a_{j} \gamma_{j}$; otherwise let $a_{1}=\cdots=a_{l-1}=0$. Let $c_{6}=1+\max _{i, j}\left|\lambda_{i, j}\right|+\max _{j}\left|a_{j}\right|$. Then the upper bound in the lemma follows from the claim

$$
H \cap \Gamma(\underline{S}) \subset \bigcup_{i=1}^{N} \beta_{i}+\left(\Gamma_{l-1}\left(c_{6} S_{1}, \ldots, c_{6} S_{l-1}\right) \cap H\right) .
$$

To prove the claim, let $\gamma=\sum_{j=1}^{l} n_{j} \gamma_{j} \in H \cap \Gamma(\underline{S})$, with $\left|n_{j}\right|<S_{j}$. There is an index $i$ such that $\gamma-\beta_{i}$ belongs to $\Gamma_{l-1}$. If $n_{l}=\lambda_{i, l}$ the claim is obvious. Otherwise $K$ is finite, and $n_{l}-\lambda_{i, l}=m K$ for some integer $m$. Then $\gamma-\beta_{i}=\sum_{j=1}^{l-1}\left(n_{j}-\lambda_{i, j}+m a_{j}\right) \gamma_{j}$ and the claim follows.

We fix a basis $\underline{\partial}=\left(\partial_{1}, \ldots, \partial_{d}\right)$ of $W$. We denote by $\mathcal{W}_{k}$ the subspace of $W$ spanned by $\partial_{1}, \ldots, \partial_{k}$ for $k \in\{0, \ldots, d\}$, with $\mathcal{W}_{0}=\{0\}$. In the next lemma, we consider a quotient $\bar{W}=W /(W \cap T H)$; we write $\bar{\delta}$ for the image in $\bar{W}$ of a vector $\delta \in W$.

Lemma 1.6. For any algebraic subgroup $H$ of $G$ there is a basis $\underline{\delta}=\left(\delta_{1}, \ldots, \delta_{d}\right)$ of $W$ such that the following hold.

(i) For any $k \in\{1, \ldots, d\}$, we have $\operatorname{Span}\left(\delta_{1}, \ldots, \delta_{k}\right)=\mathcal{W}_{k}$.

(ii) The vectors $\delta_{k}$, for $k$ such that $\operatorname{dim}\left(\left(\mathcal{W}_{k} \cap T H\right) /\left(\mathcal{W}_{k-1} \cap T H\right)\right)=1$, form a basis of $W \cap T H$.

(iii) The vectors $\bar{\delta}_{k}$, for $k$ such that $\operatorname{dim}\left(\left(\mathcal{W}_{k} \cap T H\right) /\left(\mathcal{W}_{k-1} \cap T H\right)\right)=0$, form a basis of $\bar{W}$.

(iv) For any real numbers $T_{1} \geqslant \cdots \geqslant T_{d} \geqslant 1$ we have $\mathrm{Op}_{\underline{\delta}, T_{1} / d, \ldots, T_{d} / d} \subset \mathrm{Op}_{\underline{\underline{\alpha}}, \underline{T}} \subset \mathrm{Op}_{\underline{\delta}, d T_{1},(d-1) T_{2}, \ldots, T_{d}}$ and hence

where

$$
d^{-\operatorname{dim}(W \cap T H)} \Pi \leqslant \operatorname{dim}\left(\mathrm{Op}_{W \cap T H} \cap \mathrm{Op}_{\underline{\underline{\partial}}, \underline{T}}\right) \leqslant d^{\operatorname{dim}(W \cap T H)} \Pi,
$$

$$
\Pi=\prod_{k=1}^{d} T_{k}^{\operatorname{dim}\left(\frac{\mathcal{W}_{k} \cap T H}{\mathcal{W}_{k-1} \cap T H}\right)} .
$$




\section{S. FISCHLER}

Proof. We first prove, by induction on $\operatorname{dim}(W)$, that there is a basis $\left(\delta_{1}, \ldots, \delta_{d}\right)$ such that the first three assertions hold. Let $\left(\delta_{1}, \ldots, \delta_{d-1}\right)$ be such a basis for $\mathcal{W}_{d-1}$ (with respect to $H$ ). If $\mathcal{W}_{d} \cap T H=$ $\mathcal{W}_{d-1} \cap T H$, we let $\delta_{d}=\partial_{d}$. Otherwise, we choose for $\delta_{d}$ any element of $\mathcal{W}_{d} \cap T H$ that does not belong to $\mathcal{W}_{d-1} \cap T H$.

Let us prove the last assertion now. We have $\partial_{i}=\sum_{k=1}^{i} \lambda_{i, k} \delta_{k}$ with $\lambda_{i, i} \neq 0$ for any $i \in\{1, \ldots, d\}$. Let $\sigma \in \mathbb{N}_{T}^{d}$. Then $\partial^{\sigma}=\prod_{i=1}^{d}\left(\sum_{k=1}^{i} \lambda_{i, k} \delta_{k}\right)^{\sigma_{i}}$ is a polynomial in $\delta_{1}, \ldots, \delta_{d}$ of partial degrees less than $\left.d T_{1}, \overline{(d}-1\right) T_{2}, \ldots, T_{d}$. The lemma immediately follows.

\subsection{An equivalent statement}

Thanks to Lemmas 1.3, 1.5 and 1.6 it is immediately seen that Theorem 1.1 is equivalent to the following statement.

Theorem 1.7. Let $G, \Gamma, W, \gamma_{1}, \ldots, \gamma_{l}, \partial_{1}, \ldots, \partial_{d}$, be as in $\S 1.1$. There is a positive constant $c_{7}$ with the following property. Let $S_{1} \geqslant \cdots \geqslant S_{l} \geqslant 1, T_{1} \geqslant \cdots \geqslant T_{d} \geqslant 1$ and $D \geqslant 0$ be real numbers, and $\eta \in \mathcal{F}_{\underline{S}, \underline{T}}$ be a non-zero functional which vanishes identically on $\mathcal{R}(G)_{D}$. Then there is a non-zero connected algebraic subgroup $H$ of $G$ such that

$$
\prod_{j=1}^{l} S_{j}{ }^{\mathrm{rk}\left(\frac{\Gamma_{j} \cap H}{\Gamma_{j-1} \cap H}\right)} \prod_{k=1}^{d} T_{k}^{\operatorname{dim}\left(\frac{\mathcal{W}_{k} \cap T H}{\mathcal{W}_{k-1} \cap T H}\right)} \geqslant c_{7} D^{\operatorname{dim}(H)},
$$

where $\Gamma_{j}$ is generated by $\gamma_{1}, \ldots, \gamma_{j}$ and $\mathcal{W}_{k}=\operatorname{Span}\left(\partial_{1}, \ldots, \partial_{k}\right)$.

To prove that Theorem 1.1 is equivalent to Theorem 1.7, the crucial remark is that $H$ appears only through $\operatorname{dim}(H)$, the ranks of $\Gamma_{j} \cap H$ and the dimensions of $\mathcal{W}_{k} \cap T H$. Therefore we may choose, in terms of $\gamma_{1}, \ldots, \gamma_{l}$ and $\partial_{1}, \ldots, \partial_{d}$, a finite set $\mathcal{E}$ of subgroups, and assume in Theorem 1.7 that $H$ belongs to $\mathcal{E}$.

Remark. It is possible to conjecture that Theorem 1.1 holds with some constant $c_{1}$ depending only on $G, l$ and $d$. On the contrary, Theorem 1.7 does not hold if we ask $c_{7}$ to depend only on $G, l$ and $\partial_{1}, \ldots, \partial_{d}$; this is immediately seen if $G$ is, say, an abelian variety and $\Gamma$ is the $N$-torsion part of $G(\mathbb{C})$, with $N$ large.

Now we can prove that this statement is best possible, up to the value of $c_{7}$. Let $\mathcal{E}$ be as above, and let $H \in \mathcal{E}$ be a non-zero connected algebraic subgroup of $G$ such that

$$
\prod_{j=1}^{l} S_{j}^{\mathrm{rk}\left(\frac{\Gamma_{j} \cap H}{\Gamma_{j-1} \cap H}\right)} \prod_{k=1}^{d} T_{k}^{\operatorname{dim}\left(\frac{\mathcal{W}_{k} \cap T H}{\mathcal{W}_{k-1} \cap T H}\right)} \geqslant c_{7}^{\prime} D^{\operatorname{dim}(H)} .
$$

Let $\left(\delta_{1}, \ldots, \delta_{d}\right)$ be a basis given by Lemma 1.6 , and $\underline{T}^{\prime \prime}$ be the family $\left(T_{k} / d\right)_{k \in \mathcal{K}}$, where $\mathcal{K}$ is the set of indices such that $\operatorname{dim}\left(\left(\mathcal{W}_{k} \cap T H\right) /\left(\mathcal{W}_{k-1} \cap T H\right)\right)=1$. Let $\Phi$ be the linear map which associates to $P \in \mathcal{R}(G)_{D}$ the family of values, at all points in $\Gamma(\underline{S}) \cap H$, of all derivatives of $P / X_{0}^{D}$ along $W \cap T H$ up to order $\underline{T}^{\prime \prime}$ with respect to the basis $\left(\delta_{k}\right)_{k \in \mathcal{K}}$. Then $\Phi$ can be factored through $\mathcal{R}(H)_{D}$ (which is a quotient of $\left.\mathcal{R}(G)_{D}\right)$. If the constant $c_{7}^{\prime}$ is large enough (in terms of $G, \gamma_{1}, \ldots, \gamma_{l}$ and $\partial_{1}, \ldots, \partial_{d}$ ), Lemma 1.5 allows us to compute dimensions, and show that $\Phi$ cannot be surjective. This yields (thanks to Lemma 1.3) a non-zero functional $\eta \in \mathcal{F}_{\underline{T}^{\prime \prime}}^{\Gamma(\underline{S}) \cap H, W \cap T H} \subset \mathcal{F}_{\underline{S}, \underline{T}}^{\Gamma, W}$ which vanishes identically on $\mathcal{R}(G)_{D}$.

\section{Operations on functionals}

In this section, we define the projection of a functional on a quotient ( $\S 2.1)$, and the translation (together with derivation) of a functional ( $\S 2.2$ ). The difficult point is that functionals are to be 


\section{INTERPOLATION ON ALGEBRAIC GROUPS}

evaluated on homogeneous polynomials, not on 'real' functions on $G$; and this evaluation depends on the choice of the linear form $X_{0}$.

Everything is easier in the case when $G$ is a linear commutative algebraic group. In this situation, let $\mathbb{C}[G]$ be the algebra of regular functions on $G$, and $H$ be an algebraic subgroup of $G$. The projection $\pi: G \rightarrow G / H$ sends $\Gamma$ to $\bar{\Gamma}=\Gamma /(\Gamma \cap H)$ and $W$ to $\bar{W}=W /(W \cap T H)$, and hence induces a linear map $\pi_{\mathcal{F}}$ of $\mathcal{F}=\mathcal{F}^{\Gamma, W}$ to $\overline{\mathcal{F}}=\mathcal{F}^{\bar{\Gamma}, W}$. On the other hand, composition by $\pi$ gives an injective linear map $i: \mathbb{C}[G / H] \rightarrow \mathbb{C}[G]$. Then we have $\eta(i(P))=\pi_{\mathcal{F}}(\eta)(P)$ for all $P \in \mathbb{C}[G / H]$ and $\eta \in \mathcal{F}$ : the maps $i$ and $\pi_{\mathcal{F}}$ are adjoint with respect to the bilinear products $\mathcal{F} \times \mathbb{C}[G] \rightarrow \mathbb{C}$ and $\overline{\mathcal{F}} \times \mathbb{C}[G / H] \rightarrow \mathbb{C}$.

When the algebraic group $G$ is no longer assumed to be linear, homogeneous polynomials come into play and the situation is more complicated. The map $\iota_{D^{\prime}}: \mathcal{R}(G / H)_{D^{\prime}} \rightarrow \mathcal{R}(G)_{\delta_{H} D^{\prime}}$ depends on the choice of a family $p=\left(p_{0}, \ldots, p_{M}\right)$ of homogeneous polynomials, of the same degree $\delta_{H}$, which represents $\pi$ on an open subset containing $\Gamma$. It is immediately seen that $\iota_{D^{\prime}}$ and $\pi_{\mathcal{F}}$ are not adjoint in general: for $P \in \mathcal{R}(G / H)_{D^{\prime}}$ and $\eta=\gamma \otimes 1 \in \mathcal{F}$ we have

$$
\pi_{\mathcal{F}}(\eta)(P)=\frac{P(\underline{Y})}{Y_{0}^{D^{\prime}}} \circ \pi(\gamma)=\frac{P(p(\underline{X}))}{p_{0}^{D^{\prime}}(\underline{X})}(\gamma)=\left(\frac{X_{0}^{\delta_{H}}}{p_{0}(\underline{X})}(\gamma)\right)^{D^{\prime}} \eta\left(\iota_{D^{\prime}}(P)\right) .
$$

In $\S 2.1$, an adjoint map is constructed for $\iota_{D^{\prime}}$. In $\S 2.2$, the same work is done for translations and derivations.

\subsection{Projection of a functional on a quotient}

Let $H$ be an algebraic subgroup of $G$, not necessarily connected. The quotient $G / H$ has an algebraic group structure, and hence admits an embedding in a projective space $\mathbb{P}^{M}$. With respect to this embedding, the projection $\pi: G \rightarrow G / H$ is given (on an open subset $\Omega$ that contains $\Gamma$ ) by homogeneous polynomials $p_{0}, \ldots, p_{M}$ of the same degree, say $\delta_{H}$. This means that for $\left[x_{0}: \cdots: x_{N}\right]$ in $\Omega$, not all polynomials $p_{0}, \ldots, p_{M}$ vanish at $\left(x_{0}, \ldots, x_{N}\right)$, and the point $\left[p_{0}\left(x_{0}, \ldots, x_{N}\right): \cdots\right.$ : $\left.p_{M}\left(x_{0}, \ldots, x_{N}\right)\right]$ of $G / H$ is the coset, modulo $H$, of $\left[x_{0}: \cdots: x_{N}\right]$.

Let $p(\underline{X})=\left(p_{0}(\underline{X}), \ldots, p_{M}(\underline{X})\right)$, and let $\underline{Y}=\left(Y_{0}, \ldots, Y_{M}\right)$ be the coordinates on $\mathbb{P}^{M}$. Assume that $\pi(\Gamma)$ is contained in the open set $\left\{Y_{0} \neq \overline{0}\right\}$, i.e. $p_{0}$ does not vanish at any point of $\Gamma$.

Let $P(\underline{Y})$ be a homogeneous polynomial on $G / H$. We denote by $\iota P(\underline{X})=(P \circ p)(\underline{X})=$ $P\left(p_{0}(\underline{X}), \ldots, p_{M}(\underline{X})\right)$ the homogeneous polynomial on $G$, of degree $\delta_{H} \operatorname{deg}(P)$, induced by $P$.

Let $\bar{\Gamma}$ be the image of $\Gamma$ in $G / H$, and $\bar{W}$ be the image of $W$ in $T G / T H$. Then $\bar{W}$ is canonically isomorphic to $W /(W \cap T H)$ and to $(W+T H) / T H$. For $i \in\{1, \ldots, d\}$, let $\bar{\partial}_{i}$ be the image of $\partial_{i}$ in $\bar{W}$. The derivations $\bar{\partial}_{i}$, for $i \in\{1, \ldots, d\}$, span $\bar{W}$ but are not linearly independent in general.

Let $\overline{\mathcal{F}}=\mathbb{C} \bar{\Gamma} \otimes \operatorname{Sym}(\bar{W})$ be the corresponding space of functionals on $G / H$. We have a canonical projection $\pi_{\mathcal{F}}: \mathcal{F} \rightarrow \overline{\mathcal{F}}$.

In the following, given a homogeneous polynomial $R \in \mathcal{R}(G)$, we shall need to consider (for $\eta \in \mathcal{F}$ ) the map $P \mapsto \eta(R \cdot(P \circ p))$ of $\mathcal{R}(G / H)_{D^{\prime}}$ to $\mathbb{C}$. This is the reason why the following definition is useful (see Proposition 2.2).

Definition 2.1. Let $R \in \mathcal{R}(G)_{D^{\prime \prime}}$ be a homogeneous polynomial of degree $D^{\prime \prime}$, and $D^{\prime}$ be an integer. Let $\mathcal{P}_{R, D^{\prime}}$ be the linear map of $\mathcal{F}$ to $\overline{\mathcal{F}}$ defined, for all $\gamma \in \Gamma$ and $\sigma \in \mathbb{N}^{d}$, by

$$
\mathcal{P}_{R, D^{\prime}} \mathrm{ev}_{\gamma, \sigma}=\pi_{\mathcal{F}}\left(\sum_{\nu+\mu+\tilde{\sigma}=\sigma}\left(\begin{array}{ccc} 
& \sigma & \\
\tilde{\sigma} & \mu & \nu
\end{array}\right) \partial^{\nu}\left(\frac{R(\underline{X})}{X_{0}^{D^{\prime \prime}}}\right)(\gamma) \partial^{\mu}\left(\frac{p_{0}(\underline{X})^{D^{\prime}}}{X_{0}^{D^{\prime} \delta_{H}}}\right)(\gamma) \operatorname{ev} \gamma, \tilde{\sigma}\right) .
$$




\section{S. FISCHLER}

Proposition 2.2. Let $P \in \mathcal{R}(G / H)_{D^{\prime}}$ be a homogeneous polynomial of degree $D^{\prime}, R \in \mathcal{R}(G)_{D^{\prime \prime}}$ be a homogeneous polynomial of degree $D^{\prime \prime}$ and $\eta \in \mathcal{F}$. Then, with $\iota P(\underline{X})=P\left(p_{0}(\underline{X}), \ldots, p_{M}(\underline{X})\right)$ we have

$$
\eta(R \cdot \iota P)=\mathcal{P}_{R, D^{\prime}} \eta(P) .
$$

Proof. We may assume $\eta=\mathrm{ev}_{\gamma, \sigma}$. We have

$$
\frac{\iota P(\underline{X})}{X_{0}^{D^{\prime} \delta_{H}}}=\frac{p_{0}(\underline{X})^{D^{\prime}}}{X_{0}^{D^{\prime} \delta_{H}}} \cdot\left(\left(\frac{P(\underline{Y})}{Y_{0}^{D^{\prime}}}\right) \circ \pi\right)
$$

as analytic functions on the open subset of $G(\mathbb{C})$ defined by $X_{0} \neq 0$ and $p_{0}(\underline{X}) \neq 0$. Applying $\partial^{\sigma-\nu}$ for $\nu \leqslant \sigma$ yields, thanks to the Leibniz rule,

$$
\partial^{\sigma-\nu}\left(\frac{\iota P(\underline{X})}{X_{0}^{D^{\prime} \delta_{H}}}\right)(\gamma)=\sum_{\mu+\tilde{\sigma}=\sigma-\nu}\left(\begin{array}{c}
\sigma-\nu \\
\tilde{\sigma}
\end{array}\right) \partial^{\mu}\left(\frac{p_{0}(\underline{X})^{D^{\prime}}}{X_{0}^{D^{\prime} \delta_{H}}}\right)(\gamma) \bar{\partial}^{\tilde{\sigma}}\left(\frac{P(\underline{Y})}{Y_{0}^{D^{\prime}}}\right)(\bar{\gamma}),
$$

where $\bar{\gamma}$ is the image of $\gamma$ in $G / H$. Now the result immediately follows from the equation

$$
\eta(R \cdot \iota P)=\partial^{\sigma}\left(\frac{R(\underline{X}) \iota P(\underline{X})}{X_{0}^{D^{\prime} \delta_{H}+D^{\prime \prime}}}\right)(\gamma)=\sum_{\nu \leqslant \sigma}\left(\begin{array}{c}
\sigma \\
\nu
\end{array}\right) \partial^{\sigma-\nu}\left(\frac{\iota P(\underline{X})}{X_{0}^{D^{\prime} \delta_{H}}}\right)(\gamma) \partial^{\nu}\left(\frac{R(\underline{X})}{X_{0}^{D^{\prime \prime}}}\right)(\gamma) .
$$

Remark. Taking $R=1$ in Proposition 2.2 yields $\eta(\iota P)=\mathcal{P}_{1, D^{\prime}} \eta(P)$; therefore $\mathcal{P}_{1, D^{\prime}}: \mathcal{F} \rightarrow \mathcal{F}$ and $\iota: \mathcal{R}(G / H)_{D^{\prime}} \rightarrow \mathcal{R}(G)_{\delta_{H} D^{\prime}}$ are adjoint.

Assume that $\left(\partial_{i}\right)_{i \in I^{\prime \prime}}$ is a basis of $W \cap T H$, with $I^{\prime \prime} \subset\{1, \ldots, d\}$. Then $\mathcal{F}^{\Sigma, W \cap T H}$ is equipped with a filtration coming from this basis, for any finite set $\Sigma$ contained in $\left\{X_{0} \neq 0\right\}$. Now let $I^{\prime}=\{1, \ldots, d\} \backslash I^{\prime \prime}$; then $\underline{\bar{\partial}}=\left(\bar{\partial}_{i}\right)_{i \in I^{\prime}}$ is a basis of $\bar{W}$. The functionals $\overline{\mathrm{ev}}_{\bar{\gamma}, \sigma}=\bar{\gamma} \otimes \prod_{i \in I^{\prime}} \bar{\partial}_{i}^{\sigma_{i}}$, for $\bar{\gamma} \in \bar{\Gamma}$ and $\sigma \in \mathbb{N}^{I^{\prime}}$, form a basis of $\overline{\mathcal{F}}$. The images $\bar{\gamma}_{1}, \ldots, \bar{\gamma}_{l}$ of $\gamma_{1}, \ldots, \gamma_{l}$ in $G / H$ span $\bar{\Gamma}$. We obtain therefore a filtration on $\overline{\mathcal{F}}$, for which $\overline{\mathcal{F}}_{\underline{S}, \underline{T}^{\prime}}$ is spanned by the functionals $\overline{\mathrm{ev}}_{\bar{\gamma}, \sigma}$ for $\bar{\gamma} \in \bar{\Gamma}(\underline{S})$ and $\sigma \in \mathbb{N}_{\underline{T}^{\prime}}^{I^{\prime}}$, here $\underline{T}^{\prime}=\left(T_{i}\right)_{i \in I^{\prime}}$. Obviously $\pi_{\mathcal{F}}$ and $\mathcal{P}_{R, D^{\prime}}$ send $\mathcal{F}_{\underline{S}, \underline{T}}$ to $\overline{\mathcal{F}}_{\underline{S}, \underline{T}^{\prime}}$.

The following proposition will allow us (in $\S 4$ ) to use induction on the dimension of $G$.

Proposition 2.3. Assume that $\left(\partial_{i}\right)_{i \in I^{\prime \prime}}$ is a basis of $W \cap T H$, and $I^{\prime}=\{1, \ldots, d\} \backslash I^{\prime \prime}$. Let $D, D^{\prime}$, $D^{\prime \prime}$ be integers such that $D=\delta_{H} D^{\prime}+D^{\prime \prime}$. Let $S_{1}, \ldots, S_{l}, T_{1}, \ldots, T_{d} \geqslant 1$, and assume there exists $\eta \in \mathcal{F}_{\underline{S}, \underline{T}} \backslash\{0\}$ that vanishes identically on $\mathcal{R}(G)_{D}$. Then one of the following holds:

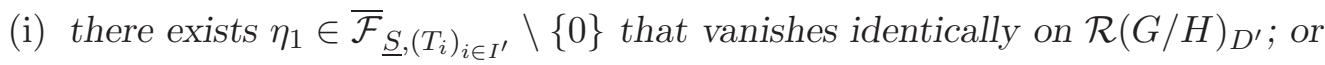

(ii) there exist $\bar{\gamma} \in \bar{\Gamma}(\underline{S})$ and $\eta_{2} \in \mathcal{F}_{\left(T_{i}\right)_{i \in I^{\prime \prime}}}^{\Gamma(S) \cap \pi^{-1}(\bar{\gamma}), W \cap T H} \backslash\{0\}$ that vanishes identically on $\mathcal{R}(G)_{D^{\prime \prime}}$.

Proof. We identify $\mathbb{N}^{d}$ to $\mathbb{N}^{I^{\prime}} \times \mathbb{N}^{I^{\prime \prime}}$. It is immediately seen that $\pi_{\mathcal{F}}\left(\mathrm{ev}_{\gamma, \sigma}\right)=\overline{\mathrm{ev}}_{\pi(\gamma), \sigma}$ if $\sigma \in$ $\mathbb{N}^{I^{\prime}} \times\{0\}^{I^{\prime \prime}}$, and $\pi_{\mathcal{F}}\left(\mathrm{ev}_{\gamma, \sigma}\right)=0$ otherwise.

Now $\eta$ satisfies $\eta(R \cdot \iota P)=\mathcal{P}_{R, D^{\prime}} \eta(P)=0$ for all $R \in \mathcal{R}(G)_{D^{\prime \prime}}$ and $P \in \mathcal{R}(G / H)_{D^{\prime}}$. If $\mathcal{P}_{R, D^{\prime}} \eta$ is non-zero for some $R$, then taking $\eta_{1}=\mathcal{P}_{R, D^{\prime}} \eta$ concludes the proof. Therefore we may assume $\mathcal{P}_{R, D^{\prime}} \eta=0$ for all $R \in \mathcal{R}(G)_{D^{\prime \prime}}$.

Let us write $\eta=\sum_{\gamma, \sigma} \lambda_{\gamma, \sigma} \mathrm{ev}_{\gamma, \sigma}$. Let $\sigma_{0} \in \mathbb{N}^{I^{\prime}}$ be of maximal length such that there exist $\gamma_{0} \in \Gamma$ and $\sigma_{1} \in \mathbb{N}^{I^{\prime \prime}}$ with $\lambda_{\gamma_{0},\left(\sigma_{0}, \sigma_{1}\right)} \neq 0$. Define

$$
\eta_{2}=\sum_{\substack{\gamma \in \Gamma \\
\gamma \equiv \gamma_{0} \bmod H}} \sum_{\nu, \mu \in \mathbb{N}^{I^{\prime \prime}}}\left(\begin{array}{c}
\nu+\mu \\
\nu
\end{array}\right) \partial^{(0, \mu)}\left(\frac{p_{0}(\underline{X})^{D^{\prime}}}{X_{0}^{D^{\prime} \delta_{H}}}\right)(\gamma) \lambda_{\gamma,\left(\sigma_{0}, \nu+\mu\right)} \operatorname{ev}_{\gamma,(0, \nu)} .
$$




\section{INTERPOLATION ON ALGEBRAIC GROUPS}

Then $\eta_{2}$ belongs to $\mathcal{F}_{\left(T_{i}\right)_{i \in I^{\prime \prime}}}^{\Gamma(S) \cap \pi^{-1}(\bar{\gamma}), W \cap T H}$ with $\bar{\gamma}=\pi\left(\gamma_{0}\right)$. Let $\sigma_{1} \in \mathbb{N}^{I^{\prime \prime}}$ be of maximal length such that $\lambda_{\gamma_{0},\left(\sigma_{0}, \sigma_{1}\right)} \neq 0$. Then the coordinate of $\eta_{2}$ on $\operatorname{ev}_{\gamma_{0},\left(0, \sigma_{1}\right)}$ is

$$
\frac{p_{0}(\underline{X})^{D^{\prime}}}{X_{0}^{D^{\prime} \delta_{H}}}(\gamma) \lambda_{\gamma,\left(\sigma_{0}, \sigma_{1}\right)} \neq 0
$$

therefore $\eta_{2} \neq 0$. Moreover, $\eta_{2}(R)$ is the coordinate of $\mathcal{P}_{R, D^{\prime}} \eta=0$ on $\overline{\mathrm{ev}}_{\bar{\gamma}, \sigma_{0}}$; accordingly it vanishes for any $R \in \mathcal{R}(G)_{D^{\prime \prime}}$.

Remark. Another proof of this proposition can be given (see [Fis03, p. 153]), which generalizes Masser's arguments (see the end of $\S 4$ in [Mas82], especially (22)).

\subsection{Translation and derivation of functionals}

Let $a$ and $b$ be integers such that there exists a complete system of addition laws on $G$ of bi-degree $(a, b)$. This means that the addition on $G$ (embedded in $\mathbb{P}^{N}$ ) is represented, on every element of a suitable open cover, by a family of bi-homogeneous polynomials of bi-degree $(a, b)$. Let $E_{0}(\underline{X}, \underline{Y}), \ldots, E_{N}(\underline{X}, \underline{Y})$ be a family of such bi-homogeneous polynomials, which represents the addition on an open subset containing $\Gamma$ (see [MW81, p. 493]); we let $\underline{X}=\left(X_{0}, \ldots, X_{N}\right)$, $\underline{Y}=\left(Y_{0}, \ldots, Y_{N}\right)$ and $\underline{E}=\left(E_{0}, \ldots, E_{N}\right)$. Assumption (1) implies that $E_{0}$ does not vanish at any point $(\gamma, \delta) \in \Gamma \times \Gamma$.

Let us consider now the algebraic group $G \times G$. We can see $W \times W$ as a subspace of $T G \times T G \simeq$ $T(G \times G)$ and $\Gamma \times \Gamma$ as a finitely generated subgroup of $(G \times G)(\mathbb{C})$. The basis $\left(\partial_{1}, \ldots, \partial_{d}\right)$ of $W$ gives $2 d$ linearly independent derivations along $W \times W$ : we denote by $\partial_{\underline{X}, i}$ the ones along $W \times\{0\}$, and by $\partial_{\underline{Y}, i}$ the ones along $\{0\} \times W$, for $i \in\{1, \ldots, d\}$. Using coordinates $(\underline{X}, \underline{Y})=\left(X_{0}, \ldots, X_{N}, Y_{0}, \ldots, Y_{N}\right)$ on $G \times G, \partial_{\underline{X}, i}$ acts only on the variables $\underline{X}$. We are now in position to apply the 'Baker-CoatesAnderson trick' in the following way.

Let $P \in \mathcal{R}(G)$ be a homogeneous polynomial of degree $D^{\prime}$; let $\tau \in \mathbb{N}^{d}$ and $\delta \in \Gamma$. We consider

$$
t_{\delta, \tau, D^{\prime}} P(\underline{X})=\partial_{\underline{Y}}^{\tau}\left(\frac{P(\underline{E}(\underline{X}, \underline{Y}))}{Y_{0}^{b D^{\prime}}}\right)(\delta),
$$

where $\partial_{\underline{Y}}^{\tau}=\partial_{\underline{Y}, 1}^{\tau_{1}} \ldots \partial_{\underline{Y}, d}^{\tau_{d}}$. The polynomial $P(\underline{E}(\underline{X}, \underline{Y}))$ is bi-homogeneous of bi-degree $\left(a D^{\prime}, b D^{\prime}\right)$ and $\partial_{\underline{Y}}^{\tau}$ does not change the degree in $\underline{X}$; therefore $t_{\delta, \tau, D^{\prime}} P(\underline{X})$ is homogeneous of degree $a D^{\prime}$. Applying $t_{\delta, \tau, D^{\prime}}$ essentially amounts to a translation by $\delta$ and a differentiation by $\partial^{\tau}$; what is important here is that the degree of $t_{\delta, \tau, D^{\prime}} P$ is controlled precisely in terms of the degree of $P$, independently of $\tau$ and $\delta$.

Proposition 2.5 below shows that the map $t_{\delta, \tau, D^{\prime}}: \mathcal{R}(G)_{D^{\prime}} \rightarrow \mathcal{R}(G)_{a D^{\prime}}$ is adjoint to the map $\mathcal{T}_{\delta, \tau, D^{\prime}}: \mathcal{F} \rightarrow \mathcal{F}$ defined by the following definition.

Definition 2.4. Let $\delta \in \Gamma, \tau \in \mathbb{N}^{d}$ and $D^{\prime}$ be an integer. We denote by $\mathcal{T}_{\delta, \tau, D^{\prime}}$ the linear map of $\mathcal{F}$ to $\mathcal{F}$ defined, for all $\gamma \in \Gamma$ and $\sigma \in \mathbb{N}^{d}$, by

$$
\mathcal{T}_{\delta, \tau, D^{\prime}} \mathrm{ev}_{\gamma, \sigma}=\sum_{\substack{0 \leqslant \tilde{\sigma} \leqslant \sigma \\
0 \leqslant \tilde{\tau} \leqslant \tau}}\left(\begin{array}{c}
\sigma \\
\tilde{\sigma}
\end{array}\right)\left(\begin{array}{l}
\tau \\
\tilde{\tau}
\end{array}\right) \partial_{\underline{X}}^{\sigma-\tilde{\sigma}} \partial_{\underline{Y}}^{\tau-\tilde{\tau}}\left(\frac{E_{0}(\underline{X}, \underline{Y})^{D^{\prime}}}{X_{0}^{a D^{\prime}} Y_{0}^{b D^{\prime}}}\right)(\gamma, \delta) \mathrm{ev}_{\gamma+\delta, \tilde{\sigma}+\tilde{\tau}}
$$

Proposition 2.5. The linear operator $\mathcal{T}_{\delta, \tau, D^{\prime}}$ is injective, and maps $\mathcal{F}_{\underline{T}}^{\Gamma(\underline{S}), W}$ to $\mathcal{F}_{\underline{T}+\tau}^{\delta+\Gamma(\underline{S}), W}$; in particular if $\delta \in \Gamma\left(\underline{S}^{\prime}\right)$ then $\mathcal{T}_{\delta, \tau, D^{\prime}} \mathcal{F}_{\underline{S}, \underline{T}}^{\Gamma, W} \subset \mathcal{F}_{\underline{S}+\underline{S^{\prime}}, \underline{T}+\tau}^{\Gamma, W}$. Moreover, for any $P \in \mathcal{R}(G)_{D^{\prime}}$ we have

$$
\eta\left(t_{\delta, \tau, D^{\prime}} P\right)=\left(\mathcal{T}_{\delta, \tau, D^{\prime}} \eta\right)(P),
$$

where $t_{\delta, \tau, D^{\prime}} P(\underline{X})$ is defined by $(4)$. 


\section{S. FISCHLER}

Proof. Let $\eta$ be a non-zero functional. Let $\sigma \in \mathbb{N}^{d}$ be of maximal length such that there exists $\gamma \in \Gamma$ with $\lambda_{\gamma, \sigma} \neq 0$. The coordinate of $\mathcal{T}_{\delta, \tau, D^{\prime}} \eta$ on $\mathrm{ev}_{\gamma+\delta, \sigma+\tau}$ is

$$
\lambda_{\gamma, \sigma} \frac{E_{0}(\underline{X}, \underline{Y})^{D^{\prime}}}{X_{0}^{a D^{\prime}} Y_{0}^{b D^{\prime}}}(\gamma, \delta) \neq 0 ;
$$

therefore the functional $\mathcal{T}_{\delta, \tau, D^{\prime} \eta}$ is non-zero. Moreover, the relation $\mathcal{T}_{\delta, \tau, D^{\prime}} \mathcal{F}_{\underline{T}}^{\Gamma(\underline{S}), W} \subset \mathcal{F}_{\underline{T}+\tau}^{\delta+\Gamma(\underline{S}), W}$ is clear. To prove that $\eta\left(t_{\delta, \tau, D^{\prime}} P\right)=\mathcal{T}_{\delta, \tau, D^{\prime}} \eta(P)$ for any $P \in \mathcal{R}(G)_{D^{\prime}}$ and $\eta \in \mathcal{F}$, we may assume $\eta=\mathrm{ev}_{\gamma, \sigma}$ and consider the equality

$$
\frac{P(\underline{E}(\underline{X}, \underline{Y}))}{X_{0}^{a D^{\prime}} Y_{0}^{b D^{\prime}}}\left(g, g^{\prime}\right)=\frac{P(Z)}{Z_{0}^{D^{\prime}}}\left(g+g^{\prime}\right) \frac{E_{0}(\underline{X}, \underline{Y})^{D^{\prime}}}{X_{0}^{a D^{\prime}} Y_{0}^{b D^{\prime}}}\left(g, g^{\prime}\right)
$$

of analytic functions of $\left(g, g^{\prime}\right)$ on the open subset of $(G \times G)(\mathbb{C})$ defined by $X_{0} \neq 0, Y_{0} \neq 0$ and $E_{0}(\underline{X}, \underline{Y}) \neq 0$. Applying $\partial_{\underline{X}, 1}^{\sigma_{1}} \ldots \partial_{\underline{X}, d}^{\sigma_{d}} \partial_{\underline{Y}, 1}^{\tau_{1}} \ldots \partial_{\underline{Y}, d}^{\tau_{d}}$ to this equality, and evaluating at the point $\left(g, g^{\prime}\right)=(\gamma, \delta)$, yields the desired result since the derivations $\partial_{i}$ commute with translations.

The special case where $\Gamma$ is torsion-free plays a special role in $\S 4$ because of the following proposition.

Proposition 2.6. Assume $\Gamma$ is torsion-free. Let $\eta \in \mathcal{F}$ be a non-zero functional, and $D^{\prime}$ be an integer. Then the functionals $\mathcal{T}_{\delta, \tau, D^{\prime}} \eta$, for $\delta \in \Gamma$ and $\tau \in \mathbb{N}^{d}$, are linearly independent.

Proof. This means that any non-trivial linear combination of the operators $\mathcal{T}_{\delta, \tau, D^{\prime}}$ (with $D^{\prime}$ fixed) is injective. Let $\eta=\sum_{\gamma, \sigma} \lambda_{\gamma, \sigma} \mathrm{ev}_{\gamma, \sigma} \in \mathcal{F} \backslash\{0\}$ and $\mathcal{T}=\sum_{\delta, \tau} \mu_{\delta, \tau} \mathcal{T}_{\delta, \tau, D^{\prime}}$, with coefficients $\mu_{\delta, \tau}$ not all zero. As $\Gamma$ is a torsion-free $\mathbb{Z}$-module of finite type, it is free; accordingly, there is a total ordering on $\Gamma \times \mathbb{N}^{d}$ compatible with addition and for which $(\gamma, \sigma)$ is greater than $(\gamma, 0)$ for any $(\gamma, \sigma) \in \Gamma \times \mathbb{N}^{d}$. Let $\left(\gamma_{0}, \sigma_{0}\right)$ be the greatest element, with respect to this ordering, such that $\lambda_{\gamma_{0}, \sigma_{0}} \neq 0$, and let $\left(\delta_{0}, \tau_{0}\right)$ be the greatest such that $\mu_{\delta_{0}, \tau_{0}} \neq 0$. Then the coordinate of $\mathcal{T} \eta$ on $\operatorname{ev}_{\gamma_{0}+\delta_{0}, \sigma_{0}+\tau_{0}}$ is

$$
\lambda_{\gamma_{0}, \sigma_{0}} \mu_{\delta_{0}, \tau_{0}} \frac{E_{0}(\underline{X}, \underline{Y})^{D^{\prime}}}{X_{0}^{a D^{\prime}} Y_{0}^{b D^{\prime}}}\left(\gamma_{0}, \delta_{0}\right) \neq 0,
$$

and hence $\mathcal{T} \eta \neq 0$.

\section{Distribution of $\left(\Gamma^{*}, \mathcal{W}\right)$}

Let $G, \Gamma$ and $W$ be as in $\S 1.1$. We let $d=\operatorname{dim}(W), n=\operatorname{dim}(G)$ and $r=\operatorname{rk}(\Gamma)$; we assume $d+r \geqslant 1$. Throughout this section, the letter $H$ always stands for a connected algebraic subgroup of $G$.

In this section, we consider subgroups $\Gamma_{1}^{*}, \ldots, \Gamma_{r}^{*}$ of $\Gamma$ such that $\{0\}=\Gamma_{0}^{*} \subset \Gamma_{1}^{*} \subset \cdots \subset \Gamma_{r}^{*}=\Gamma$ and $\operatorname{rk}\left(\Gamma_{t}^{*}\right)=t$ for any $t \in\{0, \ldots, r\}$. We write $\Gamma^{*}$ for $\Gamma$ equipped with such subgroups $\Gamma_{1}^{*}, \ldots, \Gamma_{r}^{*}$. In the same way, $\mathcal{W}$ is $W$ equipped with subspaces $\{0\}=\mathcal{W}_{0} \subset \mathcal{W}_{1} \subset \cdots \subset \mathcal{W}_{d}=W$ such that $\operatorname{dim}\left(\mathcal{W}_{k}\right)=k$ for any $k \in\{0, \ldots, d\}$. The aim of this section is to study the distribution of $\left(\Gamma^{*}, \mathcal{W}\right)$. All constructions will remain the same if $\Gamma^{*}$ is replaced by $\Gamma^{* *}$ such that $r^{\prime}=r$ and $\Gamma_{t}^{*} \cap \Gamma_{t}^{\prime *}$ is of finite index in both $\Gamma_{t}^{*}$ and $\Gamma_{t}^{\prime *}$, for any $t \in\{1, \ldots, r\}$. In $\S 3.4$, we shall associate such a pair $\left(\Gamma^{*}, \mathcal{W}\right)$ to a generating set of $\Gamma$ and a basis of $W$. All constructions (and especially names) are more transparent in this context.

\subsection{Construction and properties of the surjectivity locus}

3.1.1 Definitions. We call an obstruction to interpolation (with respect to the real parameters $\left.S_{1} \geqslant \cdots \geqslant S_{r} \geqslant 1, T_{1} \geqslant \cdots \geqslant T_{d} \geqslant 1, D\right)$ any non-zero connected algebraic subgroup $H$ of $G$ such 


\section{INTERPOLATION ON ALGEBRAIC GROUPS}

that

$$
\prod_{t=1}^{r} S_{t}^{\operatorname{rk}\left(\frac{\Gamma_{t}^{*} \cap H}{\Gamma_{t-1}^{*} \cap H}\right)} \prod_{k=1}^{d} T_{k}^{\operatorname{dim}\left(\frac{\mathcal{W}_{k} \cap T H}{\mathcal{W}_{k-1} \cap T H}\right)}>D^{\operatorname{dim}(H)} .
$$

To any connected algebraic subgroup $H$ we associate the subset $\Delta_{H}$ of $\mathbb{R}^{d+r}$ defined by the equation $\varphi_{H}(\underline{x}, \underline{y})=0$, where

$$
\varphi_{H}(\underline{x}, \underline{y})=\sum_{t=1}^{r} x_{t} \operatorname{rk}\left(\frac{\Gamma_{t}^{*} \cap H}{\Gamma_{t-1}^{*} \cap H}\right)+\sum_{k=1}^{d} y_{k} \operatorname{dim}\left(\frac{\mathcal{W}_{k} \cap T H}{\mathcal{W}_{k-1} \cap T H}\right)-\operatorname{dim}(H) .
$$

We have $\Delta_{0}=\mathbb{R}^{d+r}$, and $\Delta_{H}$ is empty if and only if $\operatorname{rk}(\Gamma \cap H)=\operatorname{dim}(W \cap T H)=0$ and $H \neq 0$. In all other cases, $\Delta_{H}$ is a hyperplane. Given a subgroup $H$, the set of all points $(\underline{x}, \underline{y}) \in \mathbb{R}_{\geqslant 0}^{d+r}$ such that $\varphi_{H}(\underline{x}, \underline{y}) \leqslant 0$ is the product of a simplex and some number (depending on $H$ ) of copies of $\mathbb{R}_{\geqslant 0}$. For $H=G$, this number is zero. Therefore the intersection of all these domains is a polytope in $\mathbb{R}^{d+r}$. This intersection is called the surjectivity locus of $\left(\Gamma^{*}, \mathcal{W}\right)$. This locus is convex (since it is an intersection of convexes). It is actually a finite intersection, since there are only finitely many functions $\varphi_{H}$ (though there might be infinitely many algebraic subgroups $H$ ). Moreover, for any $S_{1} \geqslant \cdots \geqslant S_{r} \geqslant 1, T_{1} \geqslant \cdots \geqslant T_{d} \geqslant 1$ and $D>1$, let $x_{t}=\log \left(S_{t}\right) / \log (D)$ and $y_{k}=\log \left(T_{k}\right) / \log (D)$ for $t \in\{1, \ldots, r\}$ and $k \in\{1, \ldots, d\}$. Then $(\underline{x}, \underline{y})$ belongs to the surjectivity locus of $\left(\Gamma^{*}, \mathcal{W}\right)$ if and only if there is no obstruction to interpolation with respect to $(\underline{S}, \underline{T}, D)$.

Let us denote by $\left(\mathcal{F}_{p}\right)_{p \in\{1, \ldots, M\}}$ the set of $(d+r-1)$-dimensional (closed) faces of the surjectivity locus, omitting those faces which are already faces of $\mathbb{R}_{\geqslant 0}^{d+r}$, i.e. given by the vanishing of a coordinate. For each $p \in\{1, \ldots, M\}$, let $H_{p}$ be a subgroup of $G$, of maximal dimension, such that the face $\mathcal{F}_{p}$ spans (in the affine sense) the hyperplane $\Delta_{H_{p}}$ (actually such an $H_{p}$ can be shown to be unique). This definition implies $H_{p} \neq 0$ ( since $\Delta_{0}=\mathbb{R}^{d+r}$ ). Moreover, by convexity $H_{1}, \ldots, H_{M}$ are pairwise distinct.

For $p \in\{1, \ldots, M\}$, let $\mathcal{C}_{p}$ be the cone in $\mathbb{R}_{\geqslant 0}^{d+r}$ spanned by the face $\mathcal{F}_{p}$, i.e. the set of all elements of the shape $\lambda z$ with $\lambda \in \mathbb{R}_{\geqslant 0}$ and $z \in \mathcal{F}_{p}$. Since the surjectivity locus is a convex polytope, the cones $\mathcal{C}_{1}, \ldots, \mathcal{C}_{M}$ make up a partition of $\mathbb{R}_{\geqslant 0}^{d+r}$ (up to zero-measure subsets). Moreover, since the faces $\mathcal{F}_{p}$ are convex, each cone $\mathcal{C}_{p}$ is stable under addition.

When $S_{1} \geqslant \cdots \geqslant S_{r} \geqslant 1, T_{1} \geqslant \cdots \geqslant T_{d} \geqslant 1$ and $(\log (\underline{S}), \log (\underline{T})) \in \mathcal{C}_{p_{0}}$ with $H_{p_{0}}=G,\left(\Gamma^{*}, \mathcal{W}\right)$ is said to be well distributed with respect to $(\underline{S}, \underline{T})$. In fact, when this happens, if there is an obstruction to interpolation for $(\underline{S}, \underline{T}, D)$ then $G$ itself is such an obstruction (see Proposition 3.1 below).

3.1.2 Obstructing nature of the subgroups $H_{1}, \ldots, H_{M}$. The reason why $H_{1}, \ldots, H_{M}$ and $\mathcal{C}_{1}, \ldots, \mathcal{C}_{M}$ are useful is the following.

Proposition 3.1. Let $S_{1} \geqslant \cdots \geqslant S_{r} \geqslant 1, T_{1} \geqslant \cdots \geqslant T_{d} \geqslant 1$ and $D \geqslant 0$ be real numbers such that the point $(\log (\underline{S}), \log (\underline{T}))$ belongs to $\mathcal{C}_{p}$. Let $c>0$ and $H$ be a non-zero algebraic subgroup of $G$ such that

Then we have

$$
\prod_{t=1}^{r} S_{t}^{\operatorname{rk}\left(\frac{\Gamma_{t}^{*} \cap H}{\Gamma_{t-1}^{*} \cap H}\right)} \prod_{k=1}^{d} T_{k}^{\operatorname{dim}\left(\frac{\mathcal{W}_{k} \cap T H}{\mathcal{W}_{k-1} \cap T H}\right)}>c D^{\operatorname{dim}(H)}
$$

$$
\prod_{t=1}^{r} S_{t}^{\operatorname{rk}\left(\frac{\Gamma_{t}^{*} \cap H_{p}}{\Gamma_{t-1}^{*} \cap H_{p}}\right)} \prod_{k=1}^{d} T_{k}^{\operatorname{dim}\left(\frac{\mathcal{W}_{k} \cap T H_{p}}{\mathcal{W}_{k-1} \cap T H_{p}}\right)}>c^{\operatorname{dim}\left(H_{p}\right) / \operatorname{dim}(H)} D^{\operatorname{dim}\left(H_{p}\right)} .
$$

Taking $c=1$ shows that if there is an obstruction to interpolation for $\underline{S}, \underline{T}, D$ then $H_{p}$ is such an obstruction. 


\section{S. FISCHLER}

Moreover, this proposition (along with Lemmas 1.5 and 1.6) implies that in the statement of Theorem 1.1 it is enough to assume $\#(H \cap \Gamma(\underline{S})) \operatorname{dim}\left(\mathrm{Op}_{W \cap T H} \cap \mathrm{Op} \underline{\underline{\partial}}_{\underline{T}}\right)<c_{1} D^{\operatorname{dim}(H)}$ when $H=H_{p}$ (up to a suitable change of the constant $c_{1}$ ).

Proof of Proposition 3.1. We may assume $c=1$ (by replacing $D$ by $c^{1 / \operatorname{dim}(H)} D$ ) and $D>1$. Then

$$
M=\left(\frac{\log (\underline{S})}{\log (D)}, \frac{\log (\underline{T})}{\log (D)}\right)
$$

belongs to $\mathbb{R}_{\geqslant 0}^{d+r}$ and $\varphi_{H}$ is positive at that point; accordingly $M$ does not belong to the surjectivity locus. Now $M$ belongs to $\mathcal{C}_{p}$. As the border of the surjectivity locus, in $\mathcal{C}_{p}$, is the hyperplane $\Delta_{H_{p}}$, we obtain that $\varphi_{H_{p}}$ is positive at $M$, thereby proving the proposition.

We shall also need (in the proof of Theorem 1.1) the following proposition.

Proposition 3.2. Let $S_{1} \geqslant \cdots \geqslant S_{r} \geqslant 1, T_{1} \geqslant \cdots \geqslant T_{d} \geqslant 1$ and $D \geqslant 0$ be real numbers such that the point $(\log (\underline{S}), \log (\underline{T}))$ belongs to $\mathcal{C}_{p}$. Let $H$ be a connected algebraic subgroup of $G$ which contains $H_{p}$, and is distinct from $H_{p}$. Let $c$ be a positive real number. Assume that

$$
\prod_{t=1}^{r} S_{t}^{\operatorname{rk}\left(\frac{\left(\Gamma_{t}^{*} \cap H\right) /\left(\Gamma_{t}^{*} \cap H_{p}\right)}{\left(\Gamma_{t-1}^{*} \cap H\right) /\left(\Gamma_{t-1}^{*} \cap H_{p}\right)}\right)} \prod_{k=1}^{d} T_{k}^{\operatorname{dim}\left(\frac{\left(\mathcal{W}_{k} \cap T H\right) /\left(\mathcal{W}_{k} \cap T H_{p}\right)}{\left(\mathcal{W}_{k-1} \cap T H\right) /\left(\mathcal{W}_{k-1} \cap T H_{p}\right)}\right)}>c D^{\operatorname{dim}\left(H / H_{p}\right)} .
$$

Then we have

$$
\prod_{t=1}^{r} S_{t}^{\operatorname{rk}\left(\frac{\Gamma_{t}^{*} \cap H_{p}}{\Gamma_{t-1}^{n} \cap H_{p}}\right)} \prod_{k=1}^{d} T_{k}^{\operatorname{dim}\left(\frac{\mathcal{W}_{k} \cap T H_{p}}{\mathcal{W}_{k-1} \cap T H_{p}}\right)}>c^{\operatorname{dim}\left(H_{p}\right) / \operatorname{dim}\left(H / H_{p}\right)} D^{\operatorname{dim}\left(H_{p}\right)}
$$

Proof. We may assume $c=1$ and $D>1$; then

$$
(\underline{x}, \underline{y})=\left(\frac{\log (\underline{S})}{\log (D)}, \frac{\log (\underline{T})}{\log (D)}\right)
$$

belongs to $\mathcal{C}_{p}$. The affine function $\varphi_{H}(t \underline{x}, t \underline{y})-\varphi_{H_{p}}(\underline{t} \underline{x}, \underline{y})$ is negative at $t=0$, positive at $t=1$ and non-positive at the point $t=t_{0}>0$ such that $\left(t_{0} \underline{x}, t_{0} \underline{y}\right) \in \mathcal{F}_{p}$ (since $\varphi_{H}$ is non-positive and $\varphi_{H_{p}}$ is zero at this point); therefore $t_{0}<1$. Consequently, the point $(\underline{x}, \underline{y})$ lies outside the surjectivity locus: $\varphi_{H_{p}}(\underline{x}, \underline{y})>0$. This concludes the proof of Proposition 3.2.

\subsection{Construction and properties of the injectivity locus}

In this subsection, we assume that if $H$ contains a subgroup of finite index in $\Gamma$ and $T H$ contains $W$ then $H=G$. In fact, if this is not the case, then $H$ is an obstruction to zero estimates for all parameters $\underline{S}, \underline{T}, D$, and the injectivity locus defined below is empty. Moreover, this assumption is not restrictive, since if there is such a subgroup $H$ then we may work in $H$ instead of $G$.

We call an obstruction to zero estimates (with respect to $S_{1} \geqslant \cdots \geqslant S_{r} \geqslant 1, T_{1} \geqslant \cdots \geqslant$ $\left.T_{d} \geqslant 1, D\right)$ any connected algebraic subgroup $H$ of $G$, different from $G$, such that

$$
\prod_{t=1}^{r} S_{t}^{\operatorname{rk}\left(\frac{\Gamma_{t}^{*} /\left(\Gamma_{t}^{*} \cap H\right)}{\Gamma_{t-1}^{*} /\left(\Gamma_{t-1}^{*} \cap H\right)}\right)} \prod_{k=1}^{d} T_{k}^{\operatorname{dim}\left(\frac{\mathcal{W}_{k} /\left(\mathcal{W}_{k} \cap T H\right)}{\mathcal{W}_{k-1} /\left(\mathcal{W}_{k-1} \cap T H\right)}\right)}<D^{\operatorname{dim}(G / H)}
$$

Let $\mathcal{E}_{H}$ be the set of all points $(\underline{x}, \underline{y}) \in \mathbb{R}_{\geqslant 0}^{d+r}$ such that $\left(\varphi_{G}-\varphi_{H}\right)(\underline{x}, \underline{y}) \geqslant 0$, with

$$
\left(\varphi_{G}-\varphi_{H}\right)(\underline{x}, \underline{y})=\sum_{t=1}^{r} x_{t} \operatorname{rk}\left(\frac{\Gamma_{t}^{*} /\left(\Gamma_{t}^{*} \cap H\right)}{\Gamma_{t-1}^{*} /\left(\Gamma_{t-1}^{*} \cap H\right)}\right)+\sum_{k=1}^{d} y_{k} \operatorname{dim}\left(\frac{\mathcal{W}_{k} /\left(\mathcal{W}_{k} \cap T H\right)}{\mathcal{W}_{k-1} /\left(\mathcal{W}_{k-1} \cap T H\right)}\right)-\operatorname{dim}(G / H) .
$$




\section{INTERPOLATION ON ALGEBRAIC GROUPS}

This is the complement in $\mathbb{R}_{\geqslant 0}^{d+r}$ of the product of a simplex and some number of copies of $\mathbb{R}_{\geqslant 0}$. Moreover, for $H=0$ this number is zero. We call the intersection of all these domains $\mathcal{E}_{H}$ the injectivity locus. This locus is an infinite convex polytope with finitely many closed $(d+r-1)$ dimensional faces denoted by $\left(\widetilde{\mathcal{F}}_{q}\right)_{1 \leqslant q \leqslant \widetilde{M}}$ (here we omit the faces which are already faces of $\mathbb{R}_{\geqslant 0}^{d+r}$ ).

Let $S_{1} \geqslant \cdots \geqslant S_{r} \geqslant 1, T_{1} \geqslant \cdots \geqslant T_{d} \geqslant 1$ and $D>1$ be real numbers; then

$$
\left(\frac{\log (\underline{S})}{\log (D)}, \frac{\log (\underline{T})}{\log (D)}\right)
$$

belongs to the injectivity locus if and only if there is no obstruction to zero estimates with respect to $(\underline{S}, \underline{T}, D)$.

For each $q \in\{1, \ldots, \widetilde{M}\}$, let $\widetilde{H}_{q}$ be the (unique) subgroup with minimal dimension such that the affine span of the face $\widetilde{\mathcal{F}}_{q}$ has equation $\left(\varphi_{G}-\varphi_{\widetilde{H}_{q}}\right)(\underline{x}, \underline{y})=0$. By convexity, all subgroups

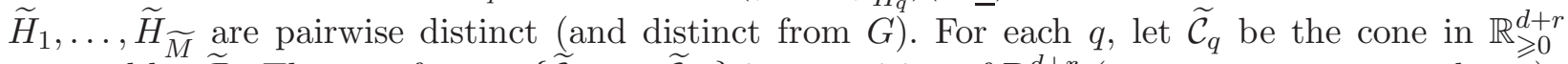
spanned by $\widetilde{\mathcal{F}}_{q}$. The set of cones $\left\{\widetilde{\mathcal{C}}_{1}, \ldots, \widetilde{\mathcal{C}}_{\widetilde{M}}\right\}$ is a partition of $\mathbb{R}_{\geqslant 0}^{d+r}$ (up to zero-measure subsets).

The following proposition means that if there is an obstruction to zero estimates for $\underline{S}, \underline{T}, D$ then some $\widetilde{H}_{q}$ is such an obstruction. Accordingly we may, in the statement of a zero estimate, assume that the obstructing subgroup is $\widetilde{H}_{q}$ (up to a change in the constant that appears in this zero estimate). This is what we shall do in $\S 3.5$, in the case where $\widetilde{H}_{q}=0$.

Proposition 3.3. Let $S_{1} \geqslant \cdots \geqslant S_{r} \geqslant 1, T_{1} \geqslant \cdots \geqslant T_{d} \geqslant 1$ and $D \geqslant 0$ be real numbers such that the point $(\log (\underline{S}), \log (\underline{T}))$ belongs to $\widetilde{\mathcal{C}}_{q}$. Let $c>0$ and $H$ be an algebraic subgroup of $G$, distinct from $G$, such that

$$
\prod_{t=1}^{r} S_{t}^{\operatorname{rk}\left(\frac{\Gamma_{t}^{*} /\left(\Gamma_{t}^{*} \cap H\right)}{\Gamma_{t-1}^{*} /\left(\Gamma_{t-1}^{*} \cap H\right)}\right)} \prod_{k=1}^{d} T_{k}^{\operatorname{dim}\left(\frac{\mathcal{W}_{k} /\left(\mathcal{W}_{k} \cap T H\right)}{\mathcal{W}_{k-1} /\left(\mathcal{W}_{k-1} \cap T H\right)}\right)}<c D^{\operatorname{dim}(G / H)}
$$

Then we have

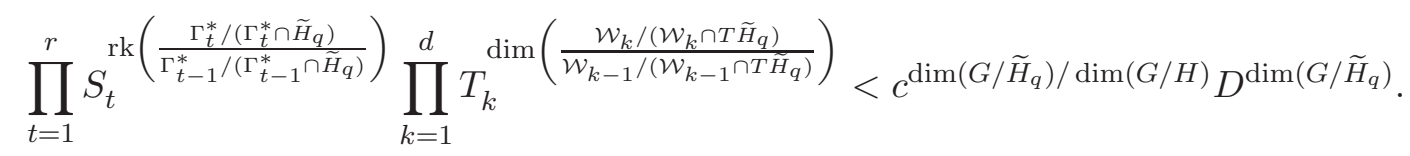

Proof. We may assume $c=1$ and $D>1$. Then the point

$$
\left(\frac{\log (\underline{S})}{\log (D)}, \frac{\log (\underline{T})}{\log (D)}\right)
$$

belongs to $\widetilde{\mathcal{C}_{q}}$ but not to the injectivity locus. Therefore $\varphi_{G}-\varphi_{\widetilde{H}_{q}}$ is negative at this point: this proves Proposition 3.3.

\subsection{Connection between injectivity and surjectivity locuses}

We have defined two decompositions of $\mathbb{R}_{\geqslant 0}^{d+r}$ into cones: one coming from interpolation ( $(3.1)$, and one from zero estimates $(\S 3.2)$. There is a connection between these decompositions, concerning the well-distributed case. To prove it, we need the following lemma.

LEMma 3.4. Let $\Delta$ be a half-line from the origin in $\mathbb{R}_{>0}^{d+r}$. Let $H$ be a connected algebraic subgroup of $G$, distinct from $G$, such that $\Delta_{H}$ is a hyperplane. Let $P_{H}, P_{G}$ and $P_{G / H}$ be the points where $\Delta$ meets $\Delta_{H}, \Delta_{G}$ and the hyperplane of equation $\left(\varphi_{G}-\varphi_{H}\right)(\underline{x}, \underline{y})=0$ (respectively). Then $P_{G}$ lies between $P_{H}$ and $P_{G / H}$. Moreover, $P_{G}$ coincides with $P_{H}$ if and only if $P_{G}$ coincides with $P_{G / H}$.

Proof. Let $(\underline{u}, \underline{v}) \in \mathbb{R}_{>0}^{d+r}$ be a non-zero vector in $\Delta$. We parametrize $\Delta$ by letting $\underline{x}=t \underline{u}$ and $\underline{y}=t \underline{v}$ for $t \in \mathbb{R}_{>0}$. We have $\varphi_{G}(\underline{x}, \underline{y})=a t-\operatorname{dim}(G)$ and $\varphi_{H}(\underline{x}, \underline{y})=a_{H} t-\operatorname{dim}(H)$ with $\bar{a}>a_{H}>0$. The points $P_{H}, P_{G}$ and $P_{G / H}$ correspond to parameters $\operatorname{dim}(H) / a_{H}, \operatorname{dim}(G) / a$ 


\section{S. FISCHLER}

and $\operatorname{dim}(G / H) /\left(a-a_{H}\right)$ (respectively). The lemma is deduced immediately from the following standard fact: if $a^{\prime}, a^{\prime \prime}, b^{\prime}, b^{\prime \prime}$ are positive real numbers then $\left(b^{\prime}+b^{\prime \prime}\right) /\left(a^{\prime}+a^{\prime \prime}\right)$ is between $b^{\prime} / a^{\prime}$ and $b^{\prime \prime} / a^{\prime \prime}$.

Proposition 3.5. There is an integer $p_{0} \in\{1, \ldots, M\}$ such that $H_{p_{0}}=G$ if and only if there is an integer $q_{0} \in\{1, \ldots, \widetilde{M}\}$ such that $\widetilde{H}_{q_{0}}=0$. When $p_{0}$ and $q_{0}$ exist, they are unique and we have $\mathcal{C}_{p_{0}}=\widetilde{\mathcal{C}}_{q_{0}}$.

Proof. Let $\Delta$ be a half-line from the origin in $\mathbb{R}_{>0}^{d+r}$, and $p_{0}$ be such that $H_{p_{0}}=G$. Then $\Delta$ belongs to the interior of $\mathcal{C}_{p_{0}}$ if and only if for any $H$ such that $\Delta_{H}$ is a hyperplane the point $P_{H}$ is not between $O$ and $P_{G}$ (where the notation is explained in Lemma 3.4). This is equivalent to the point $P_{G / H}$ being between $O$ and $P_{G}$. Now this means there is $q_{0}$ such that $\widetilde{H}_{q_{0}}=0$, and $\Delta$ belongs to the interior of $\widetilde{\mathcal{C}}_{q_{0}}$. The converse can be proved in the same way. At last, $p_{0}$ and $q_{0}$ are obviously unique, since $H_{1}, \ldots, H_{M}$ (respectively $\left.\widetilde{H}_{1}, \ldots, \widetilde{H}_{\widetilde{M}}\right)$ are pairwise distinct.

\subsection{Interpretation of both locuses}

Let us choose a generating set $\gamma=\left(\gamma_{1}, \ldots, \gamma_{l}\right)$ of $\Gamma$ and a basis $\underline{\partial}=\left(\partial_{1}, \ldots, \partial_{d}\right)$ of $W$. Then we can define $\mathcal{W}_{k}=\operatorname{Span}\left(\partial_{1}, \ldots, \overline{\partial_{k}}\right)$. Moreover let $1 \leqslant j_{1}<\cdots<\overline{j_{r}} \leqslant l$ be the indices $j$ such that $\operatorname{rk}\left(\Gamma_{j} / \Gamma_{j-1}\right)=1$, where $\Gamma_{j}$ is the subgroup generated by $\gamma_{1}, \ldots, \gamma_{j}$. Then $r=\operatorname{rk}(\Gamma)$, and we let $\Gamma_{t}^{*}=\Gamma_{j_{t}}$ for any $t \in\{1, \ldots, r\}$. This is how we associate $\left(\Gamma^{*}, \mathcal{W}\right)$ to a generating set $\underline{\gamma}=\left(\gamma_{1}, \ldots, \gamma_{l}\right)$ of $\Gamma$ and a basis $\underline{\partial}=\left(\partial_{1}, \ldots, \partial_{d}\right)$ of $W$. In $\S 4$, we shall use this remark several times: to study $\Gamma\left(S_{1}, \ldots, S_{l}\right)$ with $S_{1} \geqslant \cdots \geqslant S_{l}$, we shall apply the results in this section to $\Gamma^{*}$ with parameters $S_{j_{1}} \geqslant \cdots \geqslant S_{j_{r}}$.

Theorem 1.7 and the remarks following it give two constants $c$ and $\bar{c}$, depending only on $G, \underline{\gamma}$ and $\underline{\partial}$, with the following property. Let $S_{1} \geqslant \cdots \geqslant S_{l} \geqslant 1, T_{1} \geqslant \cdots \geqslant T_{d} \geqslant 1$ and $D>1$ be real numbers. If

$$
\left(\frac{\log (\underline{S})}{\log (D)}, \frac{\log (\underline{T})}{\log (D)}\right)
$$

belongs to the surjectivity locus then the evaluation map $\Phi_{c D}$ sending any $P \in \mathcal{R}(G)_{c D}$ to the family of values $\partial^{\sigma}\left(P / X_{0}^{c D}\right)(\gamma)$ (for $\gamma \in \Gamma(\underline{S})$ and $\sigma \in \mathbb{N}_{\underline{T}}^{d}$ ) is surjective. Moreover, if $\Phi_{\bar{c} D}$ is surjective then

$$
\left(\frac{\log (\underline{S})}{\log (D)}, \frac{\log (\underline{T})}{\log (D)}\right)
$$

belongs to the surjectivity locus.

In the same way, Philippon's zero estimate [Phi96] yields two constants $c^{\prime}$ and $\overline{c^{\prime}}$ such that

$$
\left(\frac{\log (\underline{S})}{\log (D)}, \frac{\log (\underline{T})}{\log (D)}\right)
$$

belongs to the injectivity locus if (respectively only if) $\Phi_{\overline{c^{\prime}} D}$ (respectively $\Phi_{c^{\prime} D}$ ) is injective.

\subsection{A zero estimate for well-distributed $\left(\Gamma^{*}, \mathcal{W}\right)$}

In this subsection, we write a zero estimate under the assumption that $\left(\Gamma^{*}, \mathcal{W}\right)$ is well distributed with respect to the parameters we consider. Here $\left(\Gamma^{*}, \mathcal{W}\right)$ is associated to $\Gamma, W, \gamma_{1}, \ldots, \gamma_{l}, \partial_{1}, \ldots, \partial_{d}$ as explained in $\S 3.4$.

Let us recall from $\S 2.2$ that the addition of $G$ is given, on any sufficiently small open subset of $G$, by a family of bi-homogeneous polynomials of bi-degree $(a, b)$. Moreover we denote by $\operatorname{deg}(G)$ the degree in $\mathbb{P}^{N}$ of the Zariski closure of $G$.

Theorem 3.6. Let $G, \Gamma, W, \gamma_{1}, \ldots, \gamma_{l}, \partial_{1}, \ldots, \partial_{d}$ be as in $\S 1.1$. Let $D \geqslant 0, S_{1} \geqslant \cdots \geqslant S_{l} \geqslant 1$ and $T_{1} \geqslant \cdots \geqslant T_{d} \geqslant 1$ be real numbers such that $(\log (\underline{S}), \log (\underline{T}))$ belongs to the cone $\widetilde{\mathcal{C}}_{q_{0}}$, with $\widetilde{H}_{q_{0}}=0$. 


\section{INTERPOLATION ON ALGEBRAIC GROUPS}

Let $P \in \mathcal{R}(G)_{D}$ be a non-zero homogeneous polynomial of degree $[D]$ which vanishes up to order $\underline{T}$ along $W$ at any point of $\Gamma(\underline{S})$. Then we have

$$
T_{1} \cdots T_{d} \prod_{j=1}^{l} S_{j}^{\mathrm{rk}\left(\Gamma_{j} / \Gamma_{j-1}\right)} \leqslant c_{8} D^{\operatorname{dim}(G)},
$$

with $c_{8}=\left(n^{d+r} \operatorname{deg}(G) a^{n}\right)^{n}$.

Proof. Philippon's refined zero estimate (see [Phi96, Theorem 1]) gives $H$ such that

$$
\prod_{j=1}^{l} S_{j}{ }^{\operatorname{rk}\left(\frac{\Gamma_{j} /\left(\Gamma_{j} \cap H\right)}{\Gamma_{j-1} /\left(\Gamma_{j-1} \cap H\right)}\right)} \prod_{k=1}^{d} T_{k}^{\operatorname{dim}\left(\frac{\mathcal{W}_{k} /\left(\mathcal{W}_{k} \cap T H\right)}{\mathcal{W}_{k-1} /\left(\mathcal{W}_{k-1} \cap T H\right)}\right)} \leqslant n^{d+r} \operatorname{deg}(G) a^{n} D^{\operatorname{dim}(G / H)},
$$

since the vectors $\gamma_{j}$, for $j$ such that

$$
\operatorname{rk}\left(\frac{\Gamma_{j} /\left(\Gamma_{j} \cap H\right)}{\Gamma_{j-1} /\left(\Gamma_{j-1} \cap H\right)}\right)=1
$$

have linearly independent images in $(\Gamma+H) / H$. Then Proposition 3.3 (applied to $\Gamma^{*}$, as explained in $\S 3.4)$ allows us to conclude the proof.

\section{Proof of the interpolation lemma}

In this section, we prove Theorem 1.7 (which is equivalent to Theorem 1.1) using the tools introduced in $\S \S 2$ and 3. We follow Masser's proof [Mas82]: a special 'non-degenerate' case is studied first (§ 4.1), and then the general case is derived from it $(\S 4.2)$.

Let $G, \Gamma, W, \gamma_{1}, \ldots, \gamma_{l}, \partial_{1}, \ldots, \partial_{d}$ be as in $\S 1.1$. All constants that appear in this section depend on these data. To prove Theorem 1.7, we may assume there is no connected algebraic subgroup $H$ of $G$, different from $G$, which contains a subgroup of finite index in $\Gamma$ and such that $T H$ contains $W$.

We consider the pair $\left(\Gamma^{*}, \mathcal{W}\right)$ associated to $G, \Gamma, W, \gamma_{1}, \ldots, \gamma_{l}, \partial_{1}, \ldots, \partial_{d}$ as explained in $\S 3.4$.

\subsection{A non-degenerate case}

In this subsection, we assume that $\left(\Gamma^{*}, \mathcal{W}\right)$ is well distributed with respect to the parameters we consider (as defined in $\S 3.1 .1$ ), and $\gamma_{1}, \ldots, \gamma_{l}$ are linearly independent over $\mathbb{Z}$.

Proposition 4.1. Assume $\gamma_{1}, \ldots, \gamma_{l}$ are linearly independent. Let $D \geqslant 0, S_{1} \geqslant \cdots \geqslant S_{l} \geqslant 1$ and $T_{1} \geqslant \cdots \geqslant T_{d} \geqslant 1$ be real numbers such that the point $(\log (\underline{S}), \log (\underline{T}))$ belongs to $\mathcal{C}_{p_{0}}$, with $H_{p_{0}}=G$. Let $\eta \in \mathcal{F}_{\underline{S}, \underline{T}}$ be a non-zero functional vanishing identically on $\mathcal{R}(G)_{D}$. Then

$$
S_{1} \cdots S_{l} T_{1} \cdots T_{d} \geqslant c_{9} D^{\operatorname{dim}(G)}
$$

for some constant $c_{9}$.

Remark. It is possible to prove (see [Fis03, Chapter 7]) that an admissible value for $c_{9}$ is $a^{-n}\left(c_{8}(n+1) ! 3^{d+2 l+2}(d+l) !^{2}\right)^{-d-l}$, where $c_{8}$ is the constant in Theorem 3.6.

Proof of Proposition 4.1. We may assume $d+l \geqslant 1$. Choose a point $\left(\log \left(\alpha_{1}\right), \ldots, \log \left(\alpha_{l}\right), \log \left(\beta_{1}\right)\right.$, $\left.\ldots, \log \left(\beta_{d}\right)\right)$ in $\mathcal{C}_{p_{0}} \cap \mathbb{R}_{>0}^{d+l}$ such that the least component of $\left(\alpha_{1}, \ldots, \alpha_{l}, \beta_{1}, \ldots, \beta_{d}\right)$, denoted by $\varrho$, satisfies $\varrho=c_{8} n ! 2^{d+l+2} 3^{l}$ where $c_{8}$ is the constant in Theorem 3.6.

Let $f(\underline{S}, \underline{T})$ denote the number of elements in $\Gamma(\underline{S}) \times \mathbb{N}_{\underline{T}}^{d}$, that is the dimension of $\mathcal{F}_{\underline{S}, \underline{T}}$. Let $k=f\left(\alpha_{1} S_{1}, \ldots, \alpha_{l} S_{l}, \beta_{1} T_{1}, \ldots, \beta_{d} T_{d}\right)-f\left(\left(\alpha_{1}-1\right) S_{1}, \ldots,\left(\alpha_{l}-1\right) S_{l},\left(\beta_{1}-1\right) T_{1}, \ldots,\left(\beta_{d}-1\right) T_{d}\right)$. 


\section{S. FisCHLER}

We have $f(\underline{S}, \underline{T})=\left(2\left\lceil S_{1}\right\rceil-1\right) \cdots\left(2\left\lceil S_{l}\right\rceil-1\right)\left\lceil T_{1}\right\rceil \cdots\left\lceil T_{d}\right\rceil$ and hence

$$
\begin{aligned}
k & \leqslant \prod_{j=1}^{l}\left(2 \alpha_{j}+1\right) S_{j} \prod_{k=1}^{d}\left(\beta_{k}+1\right) T_{k}-\prod_{j=1}^{l}\left(2 \alpha_{j}-3\right) S_{j} \prod_{k=1}^{d}\left(\beta_{k}-1\right) T_{k} \\
& \leqslant 2 \sum_{J \subsetneq\{1, \ldots, d+l\}}(\alpha, \beta)^{J} 3^{l} S_{1} \cdots S_{l} T_{1} \cdots T_{d} \\
& \leqslant 2^{d+l+1} 3^{l} \varrho^{-1} \alpha_{1} \cdots \alpha_{l} \beta_{1} \cdots \beta_{d} S_{1} \cdots S_{l} T_{1} \cdots T_{d},
\end{aligned}
$$

where $(\alpha, \beta)^{J}$ is the product of those coordinates of $(\alpha, \beta)$ whose indices belong to $J$.

Let $D_{0}=\left[(n ! k)^{1 / n}\right]$. If $D \leqslant a D_{0}$ then $D^{n} \leqslant a^{n} n ! k$ and Proposition 4.1 follows. Assume $D>a D_{0}$. Then the functional $\eta \in \mathcal{F}_{\underline{S}, \underline{T}}$, which vanishes identically on $\mathcal{R}(G)_{D}$, vanishes identically also on $\mathcal{R}(G)_{a D_{0}}$ (see the remark after Lemma 1.3). Let us write $1=(1, \ldots, 1), \alpha \underline{S}=\left(\alpha_{1} S_{1}, \ldots, \alpha_{l} S_{l}\right)$ and so on. For $\delta \in \Gamma\left((\alpha-1) \underline{S}\right.$ ) and $\tau \in \mathbb{N}_{(\beta-1) \underline{T}}^{d}$, let us consider $\mathcal{T}_{\delta, \tau, D_{0}} \eta$ (with the notation of $\S 2.2)$. We define in this way $f\left((\alpha-1) \underline{S}\right.$, $(\beta-\underline{1}) \underline{T}$ ) functionals, which belong to $\mathcal{F}_{\alpha \underline{S}, \beta \underline{\underline{T}}}$, are linearly independent (by Proposition 2.6), and vanish identically on $\mathcal{R}(G)_{D_{0}}$ (by Proposition 2.5). Consequently, the map $\Psi: \mathcal{F}_{\alpha \underline{S}, \beta \underline{T}} \rightarrow\left(\mathcal{R}(G)_{D_{0}}\right)^{*}$ which associates, to any functional, the linear form on $\mathcal{R}(G)_{D_{0}}$ defined by $(2)$ has a kernel of dimension at least $f((\alpha-1) \underline{S}$, $(\beta-1) \underline{T})$. Hence

$$
\operatorname{rk}(\Psi) \leqslant f(\alpha \underline{S}, \beta \underline{T})-f((\alpha-1) \underline{S},(\beta-1) \underline{T})=k<\frac{\left(D_{0}+1\right)^{n}}{n !} \leqslant\left(\begin{array}{c}
D_{0}+n \\
n
\end{array}\right) \leqslant \operatorname{dim}\left(\mathcal{R}(G)_{D_{0}}\right)^{*},
$$

where the last inequality follows from the proof of [Mas82, Lemma 2]. Therefore $\Psi$ is not surjective: the image of $\Psi$ is contained in a hyperplane, which is the orthogonal subspace to a non-zero polynomial $P \in \mathcal{R}(G)_{D_{0}}$. This polynomial vanishes up to order $\beta \underline{T}$ along $W$ at each point of $\Gamma(\alpha \underline{S})$. Now the point $(\log (\underline{S}), \log (\underline{T}))$ belongs to $\mathcal{C}_{p_{0}}=\widetilde{\mathcal{C}}_{q_{0}}$ (thanks to Proposition 3.5), and translation by $(\log (\alpha), \log (\beta))$ sends $\widetilde{\mathcal{C}}_{q_{0}}$ to itself. Therefore Theorem 3.6 gives

$$
\begin{aligned}
\alpha_{1} \cdots \alpha_{l} \beta_{1} \cdots \beta_{d} S_{1} \cdots S_{l} T_{1} \cdots T_{d} & \leqslant c_{8} D_{0}^{n} \\
& \leqslant c_{8} n ! 2^{d+l+1} 3^{l} \varrho^{-1} \alpha_{1} \cdots \alpha_{l} \beta_{1} \cdots \beta_{d} S_{1} \cdots S_{l} T_{1} \cdots T_{d} .
\end{aligned}
$$

This contradicts the definition of $\varrho$, thereby proving Proposition 4.1.

\subsection{Proof in the general case}

In this subsection, we prove Theorem 1.7; the proof goes by steps. In the first two steps, we assume that $\left(\Gamma^{*}, \mathcal{W}\right)$ is well distributed with respect to the parameters we consider (and the subgroup $H$ in the conclusion of Theorem 1.7 can then be chosen to be $G$ ). We deduce each step from the previous one (Step 0 is Proposition 4.1); of course, the value of $c_{7}$ changes at each step.

SteP 1. Theorem 1.7 holds if $\Gamma$ is torsion-free and the point $(\log (\underline{S}), \log (\underline{T}))$ belongs to $\mathcal{C}_{p_{0}}$, with $H_{p_{0}}=G$.

Proof of Step 1. Let $1 \leqslant j_{1}<\cdots<j_{r} \leqslant l$ be the indices $j$ such that $\operatorname{rk}\left(\Gamma_{j} / \Gamma_{j-1}\right)=1$. As $\Gamma$ is torsion-free, there are linearly independent vectors $\gamma_{1}^{\prime}, \ldots, \gamma_{r}^{\prime}$ such that, for each $t \in\{1, \ldots, r\}$, $\Gamma_{j_{t+1}-1}$ is contained, and of finite index, in the group generated by $\gamma_{1}^{\prime}, \ldots, \gamma_{t}^{\prime}\left(\right.$ with $\left.j_{r+1}=l+1\right)$. Let $\Gamma^{\prime}$ be the subgroup generated by $\gamma_{1}^{\prime}, \ldots, \gamma_{r}^{\prime}$. Then $\Gamma_{j} \subset \Gamma_{t_{j}}^{\prime}$ for any $j \in\{1, \ldots, l\}$, where $t_{j}$ is the integer $t \in\{0, \ldots, r\}$ such that $j_{t} \leqslant j \leqslant j_{t+1}-1$, with $j_{0}=1$. Lemma 1.4 gives a constant $c_{3}$ (independent of $\underline{S}$ ) such that $\Gamma(\underline{S}) \subset \Gamma^{\prime}\left(c_{3} \underline{S}^{\prime}\right)$ with $\underline{S}^{\prime}=\left(S_{j_{1}}, \ldots, S_{j_{r}}\right)$ (since $t_{j_{k}}=k$ for any $k \in$ $\{1, \ldots, r\})$. Therefore $\eta$ belongs to $\mathcal{F}_{c_{3} \underline{S^{\prime}, \underline{T}}}^{\Gamma^{\prime}, W} \subset \mathcal{F}_{\alpha_{1} S_{j_{1}}, \ldots, \alpha_{r} S_{j_{r}}, \beta_{1} T_{1}, \ldots, \beta_{d} T_{d}}^{\Gamma^{\prime}, W}$, where $\left(\log \left(\alpha_{1}\right), \ldots, \log \left(\alpha_{r}\right)\right.$, $\left.\log \left(\beta_{1}\right), \ldots, \log \left(\beta_{d}\right)\right)$ is a point in $\mathcal{C}_{p_{0}}$ whose least coordinate is greater than or equal to $\log \left(c_{3}\right)$. 


\section{INTERPOLATION ON ALGEBRAIC GROUPS}

Proposition 4.1 concludes the proof (since the cone $\mathcal{C}_{p_{0}}$ obtained from $\left(\Gamma^{\prime *}, \mathcal{W}\right)$ is the same as the one obtained from $\left(\Gamma^{*}, \mathcal{W}\right)$, and the sum of two points in $\mathcal{C}_{p_{0}}$ belongs to $\left.\mathcal{C}_{p_{0}}\right)$.

Now we show that the assumption about torsion is not necessary.

StEP 2. Theorem 1.7 holds if the point $(\log (\underline{S}), \log (\underline{T}))$ belongs to the cone $\mathcal{C}_{p_{0}}$, with $H_{p_{0}}=G$.

We use the following lemma, which is a 'trivial' form of an interpolation lemma.

Lemma 4.2. Let $\Sigma$ be a finite subset of $\mathbb{P}^{N}(\mathbb{C})$, contained in $\left\{X_{0} \neq 0\right\}$. Let $T_{1}, \ldots, T_{d}$ and $D$ be non-negative integers, and $\eta \in \mathcal{F}_{\underline{T}}^{\Sigma, W}$ be a non-zero functional which vanishes identically on $\mathcal{R}(G)_{D}$. Then $\# \Sigma>D$ if $d=0$, and $\left(T_{1} \frac{T}{+} \cdots+T_{d}\right) \# \Sigma>D$ otherwise.

Remark. When there is no multiplicity, this is Lemma 6 of [Mas82]. This special case is enough for proving Step 2, but multiplicities are needed in the proof of Step 3 (for the case $n=1$ in the induction).

Proof of Lemma 4.2. Let $\gamma, \delta \in \Sigma$ be distinct, with $\delta=\left[\delta_{0}: \cdots: \delta_{N}\right]$. Let $L_{\gamma, \delta}$ be a linear form in $X_{0}, \ldots, X_{N}$ which vanishes at $\delta$ and not at $\gamma$ (such a $L_{\gamma, \delta}$ may be chosen among the forms $\delta_{i} X_{j}-\delta_{j} X_{i}$, for $\left.i, j \in\{0, \ldots, N\}\right)$. If $W=\{0\}$, the polynomials $\prod_{\delta \in \Sigma \backslash\{\gamma\}} L_{\gamma, \delta}$, for $\gamma \in \Sigma$, are sufficient to prove Lemma 4.2 .

Assume $d=\operatorname{dim}(W)$ is positive. Let $\gamma \in \Sigma$ and $i \in\{1, \ldots, d\}$. Since the matrix

$$
\left[\partial_{j}\left(X_{k} / X_{0}\right)(\gamma)\right]_{1 \leqslant k \leqslant N, 1 \leqslant j \leqslant d}
$$

has rank $d$, there is a linear form $M_{\gamma, i}$ in $X_{0}, \ldots, X_{N}$ such that $\partial_{j}\left(M_{\gamma, i} / X_{0}\right)(\gamma)=\delta_{i, j}$ for all $j \in\{1, \ldots, d\}$, where $\delta_{i, j}$ is Kronecker's $\delta$ symbol. By adding to $M_{\gamma, i}$ a suitable multiple of $X_{0}$, we may assume that $M_{\gamma, i}$ vanishes at $\gamma$ (thanks to assumption (1)).

For $\gamma \in \Sigma$ and $\sigma \in \mathbb{N}_{\underline{T}}^{d}$, we consider $P_{\gamma, \sigma}=X_{0}^{|\underline{T}-\sigma|} M_{\gamma, 1}^{\sigma_{1}} \cdots M_{\gamma, d}^{\sigma_{d}} \prod_{\delta \in \Sigma \backslash\{\gamma\}} L_{\gamma, \delta}^{|\underline{T}|}$. Then $P_{\gamma, \sigma}$ is a homogeneous polynomial of degree $|\underline{T}| \# \Sigma$, vanishes up to order $\underline{T}$ along $W$ at any point of $\Sigma \backslash\{\gamma\}$ and satisfies $\partial^{\sigma}\left(P_{\gamma, \sigma} / X_{0}^{|\underline{T}| \# \Sigma}\right)(\gamma) \neq 0$ and $\partial^{\tilde{\sigma}}\left(P_{\gamma, \sigma} / X_{0}^{|\underline{T}| \# \Sigma}\right)(\gamma)=0$ for any $\tilde{\sigma} \in \mathbb{N}^{d}$ such that $\tilde{\sigma} \neq \sigma$ and $|\tilde{\sigma}| \leqslant|\sigma|$. This concludes the proof of Lemma 4.2 (see Lemma 1.3).

Proof of Step 2. Let $H$ denote the torsion part of $\Gamma$ (i.e. the set of all elements of $\Gamma$ which are torsion in $G$ ). This is a zero-dimensional algebraic subgroup of $G$, of finite order. We use the notation of $\S 2.1$. We may assume that $D$ is a multiple of $2 \delta_{H}$ and $D \geqslant 2(\# H)$, and apply Proposition 2.3 with $D^{\prime}=D /\left(2 \delta_{H}\right)$ and $D^{\prime \prime}=D / 2$. In the second case, Lemma 4.2 gives $\# H>D / 2$, and hence a contradiction. Let us assume now that $\eta_{1} \in \overline{\mathcal{F}}_{\underline{S}, \underline{T}}$ vanishes identically on $\mathcal{R}(G / H)_{D / 2 \delta_{H}}$, with $\eta_{1} \neq 0$. The surjectivity locus and the cones associated to $\left(\bar{\Gamma}^{*}, \mathcal{W}\right)$, where $\bar{\Gamma}=\Gamma / H$, are the same as the ones associated to $\left(\Gamma^{*}, \mathcal{W}\right)$. Moreover, the point $(\log (\underline{S}), \log (\underline{T}))$ belongs to the cone $\mathcal{C}_{p_{0}}$ : Step 2 can be deduced from Step 1.

STEP 3. Theorem 1.7 holds without any additional assumption.

To prove Step 3 we shall use the following lemma.

Lemma 4.3. Let $\left(\gamma_{1}, \ldots, \gamma_{l}\right)$ be a set of generators of $\Gamma$, and $\Delta$ be a subgroup of $\Gamma$. Then there is a set of generators $\left(\delta_{1}, \ldots, \delta_{l}\right)$ of $\Delta$ and a constant $c_{10}$ such that for any $S_{1} \geqslant \cdots \geqslant S_{l} \geqslant 1$ we have

$$
\Gamma(\underline{S}) \cap \Delta \subset \Delta\left(c_{10} S_{1}, \ldots, c_{10} S_{l}\right)
$$

and $\Gamma_{j} \cap \Delta=\Delta_{j}$ for any $j \in\{1, \ldots, l\}$ (here $\Delta_{j}$ and $\Delta\left(c_{10} \underline{S}\right.$ ) are understood with respect to $\left.\left(\delta_{1}, \ldots, \delta_{l}\right)\right)$. 


\section{S. FISCHLER}

Proof of Lemma 4.3. By induction on $l$, we may assume that $\delta_{1}, \ldots, \delta_{l-1}$ are generators of $\Delta_{l-1}=$ $\Delta \cap \Gamma_{l-1}$ such that $\Gamma_{l-1}\left(\underline{S}^{\prime}\right) \cap \Delta \subset \Delta_{l-1}\left(c_{11} \underline{S}^{\prime}\right)$ for any $\underline{S}^{\prime}=\left(S_{1}^{\prime}, \ldots, S_{l-1}^{\prime}\right)$. Let $M$ be a non-zero integer, with minimal absolute value, such that $\left(M \gamma_{l}+\Gamma_{l-1}\right) \cap \Delta \neq \emptyset$ (if there is no such $M$, we let $\delta_{l}=0$ and the proof is over). We choose for $\delta_{l}$ any element of $\left(M \gamma_{l}+\Gamma_{l-1}\right) \cap \Delta$, and write $\delta_{l}=\sum_{j=1}^{l} a_{j} \gamma_{j}$ with $a_{l}=M$. Then $\Delta_{l} \subset \Delta \cap \Gamma_{l}$. Now let $\gamma \in \Delta \cap \Gamma_{l}$; write $\gamma=\sum_{j=1}^{l} n_{j} \gamma_{j}$. Then $n_{l}$ is a multiple of $M$, and

$$
\gamma=\sum_{j=1}^{l-1}\left(n_{j}-\frac{n_{l} a_{j}}{M}\right) \gamma_{j}+\frac{n_{l}}{M} \delta_{l}
$$

belongs to $\Delta_{l}$. Moreover, if $\left|n_{j}\right|<S_{j}$ for any $j \in\{1, \ldots, l\}$ then we obtain $\gamma \in \Delta\left(c_{10} \underline{S}\right)$.

Proof of Step 3. We proceed by induction on the dimension $n$ of $G$. If $n=1$, Step 3 follows from Lemma 4.2. Assume Theorem 1.7 holds for any connected algebraic group $G$ of dimension at most $n-1$, and consider the subgroups $H_{p}$ defined in $\S 3$. For each $p \in\{1, \ldots, M\}$, we fix a projective embedding of $G / H_{p}$, and homogeneous polynomials of degree $\delta_{H_{p}}$ which represent the projection of $G$ to $G / H_{p}$, like in $\S 2.1$. Let $p \in\{1, \ldots, M\}$ be such that the point $(\log (\underline{S}), \log (\underline{T}))$ belongs to the cone $\mathcal{C}_{p}$. If $H_{p}=G$ we are in the special case dealt with in Step 2. Accordingly we may assume that $\operatorname{dim}\left(H_{p}\right)$ is between 1 and $n-1$ (and that $D$ is a multiple of $2 \delta_{H_{p}}$ ). After changing the basis $\left(\partial_{1}, \ldots, \partial_{d}\right)$ thanks to Lemma 1.6 if necessary, we may apply Proposition 2.3 with $D^{\prime}=D /\left(2 \delta_{H_{p}}\right)$ and $D^{\prime \prime}=D / 2$.

In the first case, by induction we apply Theorem 1.7 to $G / H_{p}, \bar{\Gamma}=\Gamma /\left(\Gamma \cap H_{p}\right)$ and $\bar{W}=$ $W /\left(W \cap T H_{p}\right)$; we obtain in this way a connected algebraic subgroup $H^{\prime} / H_{p}$ of $G / H_{p}$, where $H^{\prime}$ is a connected algebraic subgroup of $G$ which contains $H_{p}$, is distinct from $H_{p}$, and satisfies

$$
\prod_{j=1}^{l} S_{j}^{\operatorname{rk}\left(\frac{\left(\Gamma_{j} \cap H^{\prime}\right) /\left(\Gamma_{j} \cap H_{p}\right)}{\left(\Gamma_{j-1} \cap H^{\prime}\right) /\left(\Gamma_{j-1} \cap H_{p}\right)}\right)} \prod_{k=1}^{d} T_{k}^{\operatorname{dim}\left(\frac{\left(\mathcal{W}_{k} \cap T H^{\prime}\right) /\left(\mathcal{W}_{k} \cap T H_{p}\right)}{\left(\mathcal{W}_{k-1} \cap T H^{\prime}\right) /\left(\mathcal{W}_{k-1} \cap T H_{p}\right)}\right)} \geqslant c_{12} D^{\operatorname{dim}\left(H^{\prime} / H_{p}\right)} .
$$

Proposition 3.2 completes the proof of Theorem 1.7 in this case.

In the second case, there is $\alpha \in \Gamma(\underline{S})$ and a non-zero functional $\eta^{\prime} \in \mathcal{F}_{\underline{T}^{\prime \prime}}^{\Gamma(\underline{S}) \cap\left(\alpha+H_{p}\right), W \cap T H_{p}}$ which vanishes identically on $\mathcal{R}(G)_{D / 2}$, with $\underline{T}^{\prime \prime}=\left(T_{k}\right)_{k \in \mathcal{K}}$ where $\mathcal{K}$ is the set of indices $k$ such that

$$
\operatorname{dim}\left(\frac{\mathcal{W}_{k} \cap T H_{p}}{\mathcal{W}_{k-1} \cap T H_{p}}\right)=1
$$

Proposition 2.5 and Lemma 4.3 give a non-zero functional $\mathcal{T}_{-\alpha, 0, D / 2 a} \eta^{\prime} \in \mathcal{F}_{2 c_{10} \underline{S}, \underline{T^{\prime \prime}}}^{\Delta, W \cap T H_{p}}$ which vanishes identically on $\mathcal{R}(G)_{D / 2 a}$ (where $\Delta=\Gamma \cap H_{p}$ ). Applying Theorem 1.7 to $H_{p}, \underline{\Delta}$ and $W \cap T H_{p}$ gives a non-zero connected algebraic subgroup $H^{\prime}$ of $H_{p}$ such that

$$
\prod_{j=1}^{l} S_{j}^{\mathrm{rk}\left(\frac{\Delta_{j} \cap H^{\prime}}{\Delta_{j-1} \cap H^{\prime}}\right)} \prod_{k \in \mathcal{K}} T_{k}^{\operatorname{dim}\left(\frac{\mathcal{W}_{k} \cap T H^{\prime}}{\mathcal{W}_{k-1} \cap T H^{\prime}}\right)} \geqslant c_{13} D^{\operatorname{dim}\left(H^{\prime}\right)}
$$

This concludes the proof of Theorem 1.7 in this case.

\section{ACKNOWLEDGEMENTS}

The question of generalizing Masser's result was put to me by Michel Waldschmidt; this text has benefited from useful discussions with many people, including him, Pascal Autissier, Daniel Bertrand, Jacky Cresson, Patrice Philippon and especially Damien Roy. 


\section{INTERPOLATION ON ALGEBRAIC GROUPS}

\section{REFERENCES}

Ber04 D. Bertrand, Le théorème de Siegel-Shidlovsky revisité, Preprint (2004), available at http://www.institut.math.jussieu.fr/ preprints/index-2004.html.

Fis03 S. Fischler, Contributions à l'étude diophantienne des polylogarithmes et des groupes algébriques, PhD thesis, Université Paris VI (2003), available at http://theses-EN-ligne.in2p3.fr.

Har77 R. Hartshorne, Algebraic geometry, Graduate Texts in Mathematics, vol. 52 (Springer, Berlin, 1977).

Mas82 D. Masser, Interpolation on group varieties, in Approximations diophantiennes et nombres transcendants, Luminy, 1982, eds D. Bertrand and M. Waldschmidt, Progress in Mathematics, vol. 31 (Birkhäuser, Basel, 1983), 151-171.

MW81 D. Masser and G. Wüstholz, Zero estimates on group varieties I, Invent. Math. 64 (1981), 489-516.

Phi86 P. Philippon, Lemmes de zéros dans les groupes algébriques commutatifs, Bull. Soc. Math. France 114 (1986), 355-383; errata and addenda, Bull. Soc. Math. France 115 (1987), 397-398.

Phi96 P. Philippon, Nouveaux lemmes de zéros dans les groupes algébriques commutatifs, Rocky Mountain J. Math. 26 (1996), 1069-1088.

Wal79 M. Waldschmidt, Nombres transcendants et groupes algébriques, Astérisque, vol. 69-70 (Soc. Math. France, Paris, 1979).

Wal82 M. Waldschmidt, Dépendance de logarithmes dans les groupes algébriques, in Approximations diophantiennes et nombres transcendants, Luminy, 1982, eds D. Bertrand and M. Waldschmidt, Progress in Mathematics, vol. 31 (Birkhäuser, Basel, 1983), 289-328.

Wal89 M. Waldschmidt, La transformation de Fourier-Borel: une dualité en transcendance, Lecture given in Delphes, September 1989, available at http://www.math.jussieu.fr/ miw.

Wal91 M. Waldschmidt, Fonctions auxiliaires et fonctionnelles analytiques I, II, J. Anal. Math. 56 (1991), 231-254, 255-279.

Wal00 M. Waldschmidt, Diophantine approximation on linear algebraic groups: transcendence properties of the exponential function in several variables, Grundlehren Math. Wiss., vol. 326 (Springer, Berlin, 2000).

S. Fischler stephane.fischler@math.u-psud.fr

Département de Mathématiques et Applications, École Normale Supérieure, 45 rue d’Ulm, 75230 Paris cedex 05, France

Current address: Département de Mathématiques, Université Paris-Sud, Bâtiment 425, F-91405

Orsay cedex, France 